\title{
Algebraic Morava $K$-theory spectra over perfect fields
}

\author{
SIMONE BORGHESI
}

\begin{abstract}
In the paper [2] we constructed (co)homology theories on the category of smooth schemes which share some of the some of the defining properties of the (co)homology theories induced by the Morava $k$-theory spactra in classical homotopy theory. Some proofs used the topological realization functor ( $c f$. [8]). The existence of that functor requires the base field $k$ to be embedded in $\mathbb{C}$. In this manuscript we investigate up to what extent we can obtain the same results under the sole assumption of perfectness of the base field. The results proved here guarantee the existence of spectra $\boldsymbol{\Phi}_{i}$ satisfying the same properties as in [2], provided that the algebra of all the bistable motivic cohomology operations verifies an assumption involving the Milnor operation $Q_{t}$.
\end{abstract}

Mathematics Subject Classification (2000): 14F42 (primary); 55P42, 14A15 (secondary).

\section{Introduction}

Let $k$ be a field and $S m / k$ be the category of smooth schemes over $k$. In the paper [2], under the assumption that the base field is embeded in $\mathbb{C}$, we constructed a family of (co)homology theories $\boldsymbol{\Phi}_{i}$ satisfying certain properties. If $H^{*, *}(X, \mathbb{Z} / q)$ denotes the motivic cohomology groups of a smooth scheme $X$, as defined by V. Voevodsky in [10], then $\Phi_{i}^{*, *}(X)$ are $i+1$-tuple extensions of the groups $H^{*-2 j\left(q^{t}-1\right), *-j\left(q^{t}-1\right)}(X, \mathbb{Z} / q)$ which detect certain Chern numbers of the tangent bundle of $X$. The (co)homology theories $\boldsymbol{\Phi}_{i}(\quad)$ correspond to objects denoted $\boldsymbol{\Phi}_{i}$ of a certain category $\mathcal{S H}(k)$. This is called the stable homotopy category of schemes over a base field $k$, and it is strictly related to the usual stable homotopy category of topological spaces. There are two, equivalent, constructions of this category ( $c f$. [4] and [9]), each having its own advantages. If we assume the base field $k$ to be perfect, motivic cohomology is a representable functor, represented by an object $\mathbf{H}_{\mathbb{Z}} \in \mathcal{S H}(k)$, called the Eilenberg-MacLane spectrum. This is the only instance where we use perfectness of $k$. The word spectrum refers to an object of the category $\mathcal{S H}(k)$. This is the type of objects we will deal with in this manuscript. 
The purpose of the paper is to construct the spectra $\boldsymbol{\Phi}_{i}$ of [2] for a more general kind of base fields: we now only assume the base field $k$ to be perfect instead of admitting an embedding $k \hookrightarrow \mathbb{C}$. The spectra $\boldsymbol{\Phi}_{i}$ constructed in [2] depend on a prime number $q$ and on a positive integer $t$. There are two main properties these spectra have: the first is that they fit in exact triangles

$$
\Sigma^{2\left(q^{t}-1\right), q^{t}-1} \boldsymbol{\Phi}_{i-1} \longrightarrow \boldsymbol{\Phi}_{i} \stackrel{\tau_{i}}{\longrightarrow} \mathbf{H}_{\mathbb{Z} / q}
$$

thus they are assembled by using motivic Eilenberg MacLane spectra. The second is that the way these spectra are put together is nontrivial. In particular $\boldsymbol{\Phi}_{i}$ are not simply a direct sum (wedge) of suspensions of motivic Eilenberg MacLane spectra. This property follows from the definition of $\boldsymbol{\Phi}_{i}$ given in Theorem 5.1 and that the cohomology classes $y_{i} \in H^{2(i+1)\left(q^{t}-1\right)+1,(i+1)\left(q^{t}-1\right)}\left(\boldsymbol{\Phi}_{i}, \mathbb{Z} / q\right)$ that appear in the exact triangle (5.1) are chosen so that $\delta y_{i}=Q_{t} \Sigma^{2 i\left(q^{t}-1\right)+1, i\left(q^{t}-1\right)} \iota$. The program we will follow is exactly the same as the one already employed in [2]. More precisely we determine homotopy classes $a_{n}$ of the algebraic cobordism spectrum MGI that have an appropriate divisibility property with respect to their images through the Hurewicz homomorphism (Theorem 3.1). These homotopy classes are then employed to produce a spectrum $k^{\prime}(t)$ which will serve as "model" in the costruction of $\boldsymbol{\Phi}_{i}$ in the Theorem 5.1. To us the most important features of $k^{\prime}(t)$ are the existence of a canonical MGI module structure on it and its motivic cohomology with coefficients in $\mathbb{Z} / q$. To check that the motivic cohomology is as in Theorem 4.6, we first have to compute motivic cohomology groups of the spectrum MGI as module over the motivic Steenrod algebra (Theorem 4.1) and then use this answer in the argument already described in [2] to prove Theorem 12. In that paper we used the assumption that the base field $k$ admits an embedding in $\mathbb{C}$ three times, namely in the proofs of Theorem 10, Proposition 6 and few times in Section 4.3 to prove Theorem 13. In each of them the $\mathbb{C}$ realization functor ( $c f$. [8]) has been employed and its existence relies on such assumption on the base field. This paper provides alternative proofs for those statements, requiring no assumption on the base field other than its perfectness, except in Section 5. Following our approach, the existence of a spectrum with motivic cohomology isomorphic to $\mathcal{A}^{* *} / \mathcal{A}^{* *} Q_{t}$ such as $k^{\prime}(t)$ enables us to place the obstructions to the existence of $\boldsymbol{\Phi}_{i}$ in the most "exotic" part of the algebra of the bistable motivic cohomology operations, that is the kernel $\mathcal{B}^{* *}$ of the canonical map $\mathcal{A}_{m}^{* *} \rightarrow \mathcal{A}^{* *}$ (see the part following the Theorem 4.6). In Section 5 we prove that such obstructions are contained in certain homogeneous degrees of $\mathcal{B}^{* *} / \mathcal{B}^{* *} Q_{t}$ (see the condition and (5) and (5)). In Proposition 5.2 we show that the Margolis homology groups of $\mathcal{B}^{* *}$ vanish if all the motivic cohomology operations in $\mathcal{A}_{m}^{* *}$ satisfy a Cartan formula. The issue whether $\mathcal{B}^{* *}$ satisfies such conditions is quite involved. $\mathcal{B}^{* *}$ is expected to be zero for all perfect fields, in which case the conditions would be fulfilled trivially. The proof that $\mathcal{B}^{* *}$ is zero for characteristic zero fields, recently appeared in the paper [16].

We will repeatedly use the vanishing of the motivic cohomology groups $H^{i, j}(X, A)$, with coefficients in an Abelian group $A$, of a smooth scheme $X$ in 
the following range:

$$
j<0, \quad i>2 j, \quad i-j>\text { Krull dimension of } \mathrm{X}
$$

in the case the base field $k$ is perfect, there is a pair of adjoint functors

$$
D M_{\mathrm{eff}}^{-}(k) \stackrel{R}{\rightleftarrows} \mathcal{H}(k)
$$

where $D M_{\text {eff }}^{-}(k)$ is the derived category of effective motives and $\mathcal{H}(k)$ is the (unstable) homotopy category of smooth schemes. By means of this adjunction it is possible to prove that the motivic cohomology is represented by the class in $\mathcal{H}(k)$ of certain simplicial sheaves [12]. These simplicial sheaves can be assembled together to give a spectrum in $\mathcal{S H}(k)$ which represents motivic cohomology in $\mathcal{S H}(k)$, as well.

ACKNOWLEDGEMENTS. This paper has been written during my staying at the Institute des Hautes Études Scientifiques of which I have very much appreciated the welcoming and exciting environment offered to me. I would also like to thank the Scuola Normale Superiore for its support and Ignazio Longhi for having helped me to check a technical part of the paper.

\section{Notations and basic facts}

Much like the topological stable homotopy category, $\mathcal{S H}(k)$ can be seen as the homotopy category associated to two model categories: one of which has symmetric spectra as objects and the other $S$ modules in the the sense of [3] (cf. [4] and [9], respectively). The associated homotopy categories are canonically equivalent. The main difference from the topological case is that here the category $\Delta^{\mathrm{op}}\left(\operatorname{Shv}(S m / k)_{\mathrm{Nis}}\right)$ of simplicial Nisnevich sheaves of sets over the site of smooth schemes over a base field $k$ plays the role of the category of topological spaces and the former is not locally contractible and some appropriate version of the Whitehead theorem is not known, at this date. We have distinguished pointed simplicial sheaves: $S_{S}^{1}$ is the constant simplicial sheaf $\Delta^{1} / \partial \Delta^{1}$ pointed by $\partial \Delta^{1}$, where $\Delta^{n}$ is the sheaf of simplicial sets constantly equal to the simplicial set $\Delta^{n} . \mathbb{P}_{k}^{1}$ is the constant simplicial sheaf $\mathcal{U} \rightarrow \operatorname{Hom}_{S m(k)}\left(\mathcal{U}, \mathbb{P}_{k}^{1}\right)$, for all smooth schemes $\mathcal{U}$ pointed by $\mathcal{U} \rightarrow \operatorname{Hom}_{S m(k)}(\mathcal{U},\{\infty\})$ with $\infty \in \mathbb{P}_{k}^{1}$. If $\mathcal{X}$ is a pointed sheaf, then we will denote $\Sigma^{\infty} \mathcal{X}$ its suspension spectrum, that is the spectrum consisting of $\left(\mathbb{P}_{k}^{1}\right)^{\wedge i} \wedge \mathcal{X}$ at nonnegative level $i$ and identities as structure morphisms. Let $G$ be an Abelian group, then $\mathbf{H}_{G}$ is the motivic Eilenberg-MacLane spectrum with coefficients in $G$ as described in [2, Definition 3]. As usual, if $\mathbf{A}$ and $\mathbf{B}$ are spectra we define the following groups: 


\section{Definition 2.1.}

(1) $\mathbf{A}^{i, j}(\mathbf{B})=\left[\mathbf{B}, \Sigma^{\infty}\left(S_{s}^{i-2 j} \wedge\left(\mathbb{P}_{k}^{1}\right)^{\wedge j}\right) \wedge \mathbf{A}\right]_{\mathcal{S H}(k)}$,

(2) $\mathbf{A}_{i, j}(\mathbf{B})=\left[\Sigma^{\infty}\left(S_{s}^{i-2 j} \wedge\left(\mathbb{P}_{k}^{1}\right)^{\wedge j}\right), \mathbf{A} \wedge \mathbf{B}\right]_{\mathcal{S H}(k)}$

(3) $\pi_{i, j}(\mathbf{A})=\left[\Sigma^{\infty}\left(S_{s}^{i-2 j} \wedge\left(\mathbb{P}_{k}^{1}\right)^{\wedge j}\right), \mathbf{A}\right]_{\mathcal{S H}(k)}$.

We will use the following notation: $\Sigma^{\infty}\left(S_{s}^{i-2 j} \wedge\left(\mathbb{P}_{k}^{1}\right)^{\wedge j}\right) \wedge \mathbf{A}=\Sigma^{i, j} \mathbf{A}$. If the simplicial sheaf $X$ has a point $x: \operatorname{Spec} k \rightarrow X$, the group $\tilde{\mathbf{A}}^{i, j}(X)$ is defined as $\left[\Sigma^{\infty} X, \Sigma^{i, j} \mathbf{A}\right]_{\mathcal{S H}(k)}$ with $X$ pointed by $x$. Similarly, we define $\tilde{\mathbf{A}}_{i, j}(X)$. In particular, $H^{i, j}(X, G)$ denotes the group $\left[\Sigma^{\infty} X_{+}, \Sigma^{i, j} \mathbf{H}_{G}\right]$. If $\mathbf{E}$ is a ring spectrum, $\tilde{\mathbf{A}}^{i, j}(\mathbf{E})$ is the kernel of the map $u^{*}: \mathbf{A}^{i, j}(\mathbf{E}) \rightarrow \mathbf{A}^{i, j}\left(\mathbf{S}^{\mathbf{0}}\right)$ induced by the unit $u: \mathbf{S}^{\mathbf{0}} \rightarrow \mathbf{E}$. We recall that the spectrum MGl is constructed levelwise by means of Thom spaces exactly in the same way as it is done in the classical stable homotopy category. It has a strict multiplication satisfying the axioms making it a ring spectrum [9] and it is oriented in the sense that there exists a class $c \in \widetilde{\mathbf{M G l}} \mathbf{l}^{2,1}\left(\mathbb{P}_{k}^{\infty}\right)$ pulling back to the canonical class in $\widetilde{\mathbf{M G l}} \mathbf{l}^{2,1}\left(\mathbb{P}_{k}^{1}\right)$ via the canonical inclusion $\mathbb{P}_{k}^{1} \hookrightarrow \mathbb{P}_{k}^{\infty}$. Since its proof is valid in complete generality ( $c f$. [2, Corollary 1]), we will freely use the Thom isomorphism theorem:

Theorem 2.2. Let $\mathbf{E}$ be an oriented ring spectrum, $X$ be a connected smooth scheme and $v \rightarrow X$ be an dimensional vector bundle over $X$. Then we have isomorphisms $\mathbf{E}^{i-2 n, j-n}(X) \stackrel{\cong}{\rightrightarrows} \tilde{\mathbf{E}}^{i, j}(\mathrm{Th}(v))$ for all $i$ and $j$. More precisely, $\mathbb{P}\left(v \times \mathbb{A}^{1}\right) \stackrel{p}{\rightarrow}$ $X$ and $\mathbb{P}\left(v \times \mathbb{A}^{1}\right) \stackrel{q}{\rightarrow} \mathrm{Th}(v)$ induce a commutative diagram

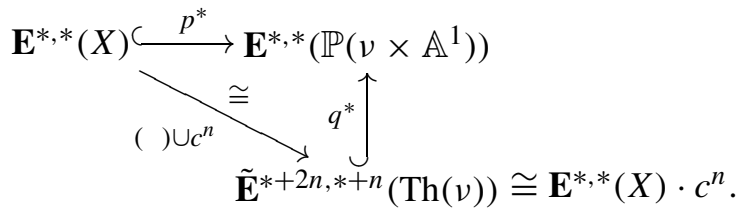

Moreover, we will regularly refer to the properties of the motivic Steenrod algebra listed in [2] and proved in [13].

\section{Choice of homotopy classes of MGI}

Our first goal is to construct an MGl module spectrum $k^{\prime}(t)$ with motivic cohomology as stated in Theorem 4.6. This can be achieved by starting with the spectrum MGI and then "killing" the homotopy classes of MGl which we are going to describe in this section. The existence of these special homotopy classes is established by the following result

Theorem 3.1. Let $h: \pi_{*, *}(\mathbf{M G l}) \rightarrow H_{*, *}(\mathbf{M G l}, \mathbb{Z})$ be the Hurewicz homomorphism, defined by associating to $\phi: \Sigma^{r, s}\left(\mathbb{P}_{k}^{1}\right) \rightarrow$ MGl the composition $\Sigma^{r, s}\left(\mathbb{P}_{k}^{1}\right) \cong$ 
$\mathbf{S}^{0} \wedge \Sigma^{r, s}\left(\mathbb{P}_{k}^{1}\right) \stackrel{u \wedge \phi}{\rightarrow} \mathbf{H}_{\mathbb{Z}} \wedge \mathbf{M G l}$, where $u: \mathbf{S}^{0} \rightarrow \mathbf{H}_{\mathbb{Z}}$ is the unit of the canonical ring structure of $\mathbf{H}_{\mathbb{Z}}$. Then there exist classes $a_{n} \in \pi_{2 n, n}$ (MGl) for $n=0,1,2, \cdots$ such that

(1) if $n=q^{t}-1$ for some prime number $q$ and positive integer $t$ then $h\left(a_{n}\right)$ is divisible by $q$, but not by $q^{2}$;

(2) in all the other cases no prime number $q$ divides $h\left(a_{n}\right)$.

Proof. The strategy is to find homotopy classes in a way that the coefficients of their images through the Hurewicz homomorphism do not depend on the base field $k$. More precisely we will show that for certain homotopy classes $a_{n}$, the coefficients of $h\left(a_{n}\right)$ are the degree of the 0 cycle represented by homogeneous polynomials in the Chern classes of the tangent bundle of some smooth projective algebraic varieties. Such polynomials have constant integral coefficients making the coefficients of $h\left(a_{n}\right)$ independent on the base field on which these varieties are definable. In turn, these projective algebraic varieties can be taken to be disjoint unions of smooth degree $q$ hypersurfaces of dimension $q^{t}-1$ for all prime numbers $q$, and degree $(1,1)$ smooth hypersurfaces in $\mathbb{P}_{k}^{n} \times \mathbb{P}_{k}^{m}$ for appropriate $n$ and $m$ thus they can be defined on any field $k$. We are going to employ the [14, Theorem 2.11].

Theorem 3.2 (Voevodsky). Let $M$ be a connected smooth projective variety of pure dimension $d$ over a field $k$. Then there exists an integer $n$ and a vector bundle $V$ of rank $n$ on $M$ such that:

(1) $V+T_{M}=\mathcal{O}_{M}^{n+d}$, where $T_{M}$ is the tangent bundle on $M$;

(2) Let $V$ as above. Then there exists a morphism

$$
f_{V}:\left(\mathbb{P}_{k}^{1}\right)^{\wedge n+d} \rightarrow \mathrm{Th}_{M}(V)
$$

in the category $\mathcal{H}_{\bullet}(k)$ with the property that, if $k$ is perfect, the composition

$$
\begin{aligned}
H^{2 d, d}(M, \mathbb{Z}) & \stackrel{t^{\prime}}{\rightarrow} \tilde{H}^{2(n+d), n+d}\left(\operatorname{Th}_{M}(V), \mathbb{Z}\right) \stackrel{f_{V}^{*}}{\rightarrow} \\
& \rightarrow \tilde{H}^{2(n+d), n+d}\left(\left(\mathbb{P}_{k}^{1}\right)^{\wedge n+d}, \mathbb{Z}\right) \cong \mathbb{Z}
\end{aligned}
$$

can be described as sending a zero cycle

$$
\sum_{i} m_{i}\left[\operatorname{Spec} L_{i}\right] \in C H^{d}(M) \cong H^{2 d, d}(M, \mathbb{Z})
$$

to the number $\sum_{i} m_{i}\left[L_{i}: k\right]$, known in the literature as the degree of the zero cycle in question. Here, $t^{\prime}$ is the cohomological Thom isomorphism (see [2]) and the last is the (stable) suspension isomorphism. 
We introduce the following notation:

- let $M$ is a smooth projective variety of pure dimension $d$ and $V$ one of the vector bundles whose existence is asserted by the previous theorem,

- $\mathrm{Gr}_{n}$ is the infinite Grassmannian of $n$ dimensional planes,

- $v: M \rightarrow \mathrm{Gr}_{n}$ is the map in $\mathcal{H}(k)$ classifying $V$,

- $t$ is the homology Thom isomorphism,

- $i: \mathrm{Gr}_{d} \rightarrow \mathrm{Gr}:=\lim _{h} \mathrm{Gr}_{h}$ is the canonical map,

- and $j: \Sigma^{\infty} M G l_{n} \rightarrow \Sigma^{2 n, n}$ MGI the composition in $\mathcal{S H}(k)$

$$
\Sigma^{\infty} M G l_{n} \rightarrow \operatorname{MGl}[n] \stackrel{\sigma^{-n}}{\rightarrow} \Sigma^{2 n, n} \mathbf{M G l}
$$

where $M G l[n]_{i}=M G l_{i+n}(c f .[4$, Lemma 3.19]).

To each such $M$, we can associate the homotopy class $a_{d}^{V} \in \pi_{2 d, d}$ (MGI), represented by the composition in $\mathcal{S H}(k)$

$$
\Sigma^{\infty}\left(\mathbb{P}_{k}^{1}\right)^{\wedge n+d} \stackrel{f_{V}}{\rightarrow} \Sigma^{\infty} \mathrm{Th}(V) \stackrel{\operatorname{Th}(v)}{\rightarrow} \Sigma^{\infty} M G L_{n} \stackrel{j}{\rightarrow} \Sigma^{2 n, n} \text { MGl. }
$$

Essentially by definition we have that $j_{*} \circ \operatorname{Th}(v)_{*} \circ\left(f_{V}\right)_{*}\left(\Sigma^{2(n+d), n+d} \iota\right)=h\left(a_{d}^{M}\right)$ where $\iota$ is the generator of $\tilde{H}_{2(n+d), n+d}\left(\left(\mathbb{P}_{k}^{1}\right)^{\wedge n+d}, \mathbb{Z}\right)$. We will now show that the coefficients of $h\left(a_{d}^{M}\right)$ are the degrees of the zero cycles represented by certain polynomials of degree $d$ in the Chern classes. Consider the following diagram:

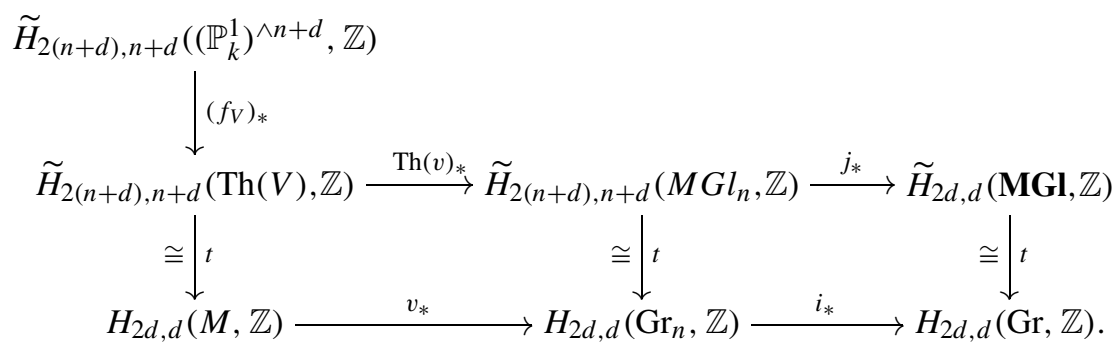

The diagram commutes by naturality of Thom isomorphism (vertical maps). In Section 4, Step 3, we will show that we can choose canonical polynomial generators $\beta_{u}$ and $b_{u}$ of the motivic homology of MGI and Gr respectively, with the property that the homological Thom isomorphism $t$ is described by

$$
\begin{aligned}
\tilde{H}_{*, *}(\mathbf{M G l}, \mathbb{Z}) & \cong H_{*, *}(\operatorname{Spec} k, \mathbb{Z})\left[\beta_{1}, \beta_{2}, \cdots\right] \stackrel{t}{\rightarrow} \\
& \rightarrow H_{*, *}(\operatorname{Spec} k, \mathbb{Z})\left[b_{1}, b_{2}, \cdots\right] \cong H_{*, *}(\mathrm{Gr}, \mathbb{Z})
\end{aligned}
$$

with $t\left(\beta_{1}^{\alpha_{1}} \cdots \beta_{r}^{\alpha_{r}}\right)=b_{1}^{\alpha_{1}} \cdots b_{r}^{\alpha_{r}}$. Therefore the coefficients of $h\left(a_{d}^{M}\right)$ can be computed via the Kronecker products $\left\langle(i \circ v)_{*}\left(t\left(\left(f_{V}\right)_{*} \iota\right)\right), s_{\alpha}\right\rangle$ having denoted $s_{\alpha}$ the 
dual class to $b_{1}^{\alpha_{1}} \cdots b_{r}^{\alpha_{r}}$ with $\alpha=\left(\alpha_{1}, \cdots, \alpha_{r}\right)$. By adjunction, this is the same as $\left\langle\left(t\left(\left(f_{V}\right)_{*} \iota\right),(i \circ v)^{*}\left(s_{\alpha}\right)\right\rangle\right.$ or even $\left\langle\iota, f_{V}^{*} t^{\prime}(i \circ v)^{*}\left(s_{\alpha}\right)\right\rangle$ having set $t^{\prime}$ to be the cohomological Thom isomorphism. Because of Theorem 3.2, $f_{V}^{*} t^{\prime}(i \circ v)^{*}\left(s_{\alpha}\right)$ is precisely the degree of the zero cycle $(i \circ v)^{*}\left(s_{\alpha}\right)$ in $M$. This is some homogeneous polynomial of degree $d$ in the Chern classes of $V$. On the other hand, since any such vector bundle $V$ satisfies the equation $T_{M} \oplus V=\mathcal{O}_{M}^{n(V)+d}$, we see inductively that the Chern classes of all these $V$ are the same and can be expressed in fuction of Chern classes of $T_{M}$. To prove Theorem 3.1.1. it suffices to present smooth projective varieties $M^{q^{t}-1}$ of dimension $q^{t}-1$ such that $q$ divides all the Chern classes $c_{k}\left(T_{M^{q^{t}-1}}\right)$, but $q^{2}$ does not divide $\operatorname{deg}\left((i \circ v)^{*}\left(s_{\alpha}\right)\right)$ for $\alpha=\left(0, \cdots, q^{t}-1\right)$, where the latter notation stands for a string of zeroes with a 1 placed in the $\left(q^{t}-1\right)-t h$ position. Notice that $s_{r} \stackrel{\text { def }}{=} s_{(0, \cdots, 1)}$ are primitive classes, therefore they are additive in the sense that if $V$ and $W$ are vector bundles, $s_{r}(V \oplus W)=s_{r}(V)+s_{r}(W)$. In particular $s_{r}\left(T_{M}\right)=-s_{r}(V)$. We claim that any degree $q$ smooth projective hypersurface in $\mathbb{P}^{q^{t}}$ can be taken to be $M^{q^{t}-1}$. In general, if $i: M \hookrightarrow \mathbb{P}^{N}$ is an hypersurface, we have that $i^{*} x=x \cup c_{1}(\mathcal{O}(M))$ for any cycle $x \in C H^{*}\left(\mathbb{P}^{N}\right)$. If $M$ is of degree $h$, then $c_{1}(\mathcal{O}(M))=h \cdot L$, where $L$ is the class of an hyperplane. On the other hand, we have an exact sequence of vector bundles:

$$
0 \rightarrow T_{M} \rightarrow i^{*} T_{\mathbb{P}^{N}} \rightarrow i^{*} \mathcal{O}(M) \rightarrow 0
$$

yielding $c_{k}\left(T_{M}\right)=c_{k}\left(i^{*} T_{\mathbb{P}^{N}}\right)-c_{k-1}\left(T_{M}\right) c_{1}\left(i^{*} \mathcal{O}(M)\right)$ for any $k$. By naturality of Chern classes, $c_{k}\left(T_{M}\right)=i^{*} c_{k}\left(T_{\mathbb{P}^{N}}\right)-c_{k-1}\left(T_{M}\right) i^{*} c_{1}(\mathcal{O}(M))$ so that $h$ divides $c_{k}\left(T_{M}\right)$ and any polynomial in the Chern classes of the tangent bundle of $M$. In particular $h$ divides all the $(i \circ v)^{*}\left(s_{\alpha}\right)$. For any positive integer $r, s_{r}\left(T_{M}\right)$ is computed by using that this type of class is additive and the answer is $s_{d}\left(T_{M}\right)=h\left(d+2-h^{d}\right)$ for a smooth hypersurface $M$ of dimension $d$ and degree $h$ (see [7, Problem 16D], for instance). If we take $h=q$ and dimension $d=q^{t}-1$ we have that $q^{2}$ does not divide $s_{d}(M)$. This proves part (3.1) of Theorem 3.1. To settle part (5.13) we argue as follows: let $H_{n, m}$ be a smooth hypersurface of degree $(1,1)$ in $\mathbb{P}^{n} \times \mathbb{P}^{m}$. Then ([7, Problem 16E])

$$
s_{n+m-1}\left(T_{H_{n, m}}\right)=\frac{-(n+m) !}{n ! m !} .
$$

If $u \neq q^{t}-1$ for some prime number $q$ and positive integer $t$, let $\alpha$ be an integer such that $\alpha q<u+1 \leq(\alpha+1) q$. We can check that

$$
s_{u}\left(H_{(u+1-\alpha q), \alpha q}\right)=\frac{(u+1) !}{(u+1-\alpha q) ! \alpha q !}
$$

is not divisible by $q$. Let now $H_{n\left(q_{i}\right), m\left(q_{i}\right)}=H_{\left(u+1-\alpha q_{i}\right), \alpha q_{i}}$ for each prime number $q_{i}<u+1$. Then, there exist integers $\lambda_{1}, \cdots \lambda_{\Phi(u)}$ so that

$$
\lambda_{1} s_{u}\left(H_{n(2), m(2)}\right)+\lambda_{2} s_{u}\left(H_{n(3), m(3)}\right)+\cdots \lambda_{\Phi(u)} s_{u}\left(H_{n(p), m(p)}\right)=1
$$


where $\Phi(u)$ is the number of the prime numbers less that $u$ and $p$ is the greatest such prime. It follows that the homotopy class

$$
a_{u}:=\sum_{i=1}^{\Phi(u)} \lambda_{i} a_{u}^{H_{n\left(q_{i}\right), m\left(q_{i}\right)}}
$$

has the property that the coefficient of $b_{u}$ in $h\left(a_{u}\right)$ equals 1 , hence $q$ does not divide $h\left(a_{u}\right)$, as sought.

\section{Motivic cohomology of MGI}

Theorem 3.1 gives us the classes necessary to proceed with the construction of the spectrum that has been denoted with $k^{\prime}(t)$ in [2]. We fix a prime number $q$ and recall that $k^{\prime}(t)$ has been defined in [2] as the homotopy colimit of the diagram

$$
\text { MGl } \stackrel{\eta_{0}}{\longrightarrow} \mathbf{E}_{0} \stackrel{\eta_{1}}{\longrightarrow} \mathbf{E}_{1} \stackrel{\eta_{2}}{\longrightarrow} \cdots
$$

with $\mathbf{E}_{i}$ defined inductively by the exact triangle

$$
\Sigma^{2 i, i} \mathbf{E}_{i-1} \stackrel{\cdot a_{i}}{\rightarrow} \mathbf{E}_{i-1} \stackrel{\eta_{i}}{\rightarrow} \mathbf{E}_{i}
$$

where $\cdot a_{i}$ is the map induced by the MGl-module multiplication by $a_{i}:\left(\mathbb{P}_{k}^{1}\right)^{\wedge i} \wedge$ $\mathbf{E}_{i-1} \stackrel{a_{i} \wedge i d}{\rightarrow}$ MGl $\wedge \mathbf{E}_{i-1} \stackrel{m}{\rightarrow} \mathbf{E}_{i-1}$. The morphism $\cdot a_{0}$ is taken to be the multiplication by $q$. Here we use that the category of MGI modules spectra is closed under homotopy colimits. In classical homotopy theory we can prove that such $k^{\prime}(t)$ is the homotopy limit of a tower of fibrations whose fibers are appropriate suspensions of Eilenberg-MacLane spectra with coefficients in $\mathbb{Z} / q$. Our purpose here is to build such tower and then we will take its homotopy limit as our model for $k(t)$. This spectra have been employed to prove the so called higher degree formulas ( $c f$. [1]) and for that it was essential to have motivic Eilenberg-MacLane spectra appearing in the tower (see also [2, page 411]). The purpose of this section is to compute the motivic cohomology of the spectra $k^{\prime}(t)$. It will turn out that it is "as expected" (Theorem 4.6). This result will be extensively used in the next section's computations. We start by expressing the motivic cohomology of MGI as graded left module over the ring $\mathcal{A}^{*, *}$. This is the sub left $H^{*, *}(\operatorname{Spec} k, \mathbb{Z} / q)$ module generated by the motivic Steenrod operations $\beta, P^{i}$ defined in [13]. It happens to be closed under the multiplication and comultiplication of $\mathcal{A}_{m}^{*, *}:=\left[\mathbf{H}_{\mathbb{Z} / q}, \Sigma^{*, *} \mathbf{H}_{\mathbb{Z} / q}\right]_{\mathcal{S H}(k)}$ so it inherits these structures. As usual we will denote $H^{*, *}(\operatorname{Spec} k, \mathbb{Z} / q)=: H^{*, *}$. In [13] V. Voevodsky has shown that the motivic Steenrod algebra $\mathcal{A}^{* *}$ is isomorphic to $H^{*, *} \otimes_{\mathbb{Z} / q} \mathcal{A}_{\text {top }}^{*, *}$ as left $H^{*, *}$ module. As $\mathbb{Z} / q$ vector space, $\mathcal{A}_{\text {top }}^{*, *}$ is generated by the operations $Q_{0}^{\epsilon_{0}} Q_{1}^{\epsilon_{1}} \cdots Q_{m}^{\epsilon_{m}}\left(r_{1}, \cdots, r_{n}\right)$ for nonnegative integers $r_{i}$ and $\epsilon_{j} \in\{0,1\}$ for all $j$. These operations are entirely analogous to their topological counterparts 
except for the bidegree and that at the prime $q=2$, the comultiplication is more involved than the usual one. We recall that the dual of the motivic Steenrod algebra $\mathcal{A}_{*, *}$ is isomorphic to a quotient of

$$
H_{*, *}\left[\xi_{1}, \xi_{2}, \cdots\right] \otimes \Lambda_{H_{*, *}}\left[\tau_{0}, \tau_{1}, \cdots\right]
$$

The operations $Q_{t}$ and $\left(r_{1}, \cdots, r_{n}\right)$ are defined as the duals to the classes $\tau_{t}$ and $\xi_{1}^{r_{1}} \cdots \xi_{n}^{r_{n}}$ and have bidegree $\left(2 q^{t}-1, q^{t}-1\right)$ and

$$
\left(\sum_{i=1}^{n} 2 r_{i}\left(q^{i}-1\right), \sum_{i=1}^{n} r_{i}\left(q^{i}-1\right)\right)
$$

respectively. For more details we refer to [13].

Theorem 4.1. For a fixed prime number $q$, denote by $T(q)$ the set of integers $\left\{q^{n}-\right.$ $\left.1: n \in \mathbb{Z}^{+}\right\}$, where $\mathbb{Z}^{+}$is the set of positive integers. Let $I=\left(i_{1}, \cdots, i_{k}\right)$ with $i_{j} \geq 0$ and $i_{j} \in \mathbb{Z}^{+}-T(q)$. Then $H^{* * *}(\mathbf{M G l}, \mathbb{Z} / q)$ is isomorphic to

$$
\frac{\mathcal{A}^{* *}}{\mathcal{A}^{* *}\left(Q_{0}, \cdots, Q_{t}, \cdots\right)} \cdot\left\{d_{I}^{\prime}, I=\left(i_{1}, \cdots, i_{k}\right)\right\}
$$

as graded left $\mathcal{A}^{* *}$ module. The basis elements $d_{I}^{\prime}$ are assigned the bidegree $\left(2\left(\sum_{j} j i_{j}\right), \sum_{j} j i_{j}\right)$.

Remark 4.2. Using the commuting rules of the [2, Corollary 4], we see that the $H^{*, *}$ subalgebra of $\mathcal{A}^{* *}$ generated by $\left\{\left(r_{1}, \cdots, r_{n}\right)\right\}$ for all $r_{i} \geq 0$ is isomorphic, as left $H^{*, *}$ module, to $\frac{\mathcal{A}^{* *}}{\mathcal{A}^{* *}\left(Q_{0}, \cdots, Q_{t}, \cdots\right)}$, although it may happen that $\frac{\mathcal{A}^{* *}}{\mathcal{A}^{* *}\left(Q_{0}, \cdots, Q_{t}, \cdots\right)}$ is not a ring (for instance if $q=2, \sqrt{-1} \notin k$ and char $(k) \neq 2$, simultaneously).

Proof. The statement will follow by duality, from:

Theorem 4.3. For any prime number $q$, there is a left comodule algebra isomorphisms over the dual motivic Steenrod algebra $\mathcal{A}_{*, *}$ :

$$
\tilde{H}_{*, *}(\mathbf{M G l}, \mathbb{Z} / q) \cong H_{*, *}\left[\xi_{1}, \cdots, \xi_{n}, \cdots\right] \otimes_{H_{*, *}} H_{*, *}\left[d_{i}, i \neq q^{n}-1, \forall n\right]
$$

with $\left|d_{i}\right|=(2 i, i)$ and $H_{*, *}\left[\xi_{1}, \cdots, \xi_{n}, \cdots\right] \hookrightarrow \mathcal{A}^{* *}$. The $\mathcal{A}_{*, *}$ comodule structure of the tensor product is $\psi\left(p\left(\xi_{i}\right) \otimes r\left(d_{j}\right)\right)=\psi\left(p\left(\xi_{i}\right)\right) \otimes r\left(d_{j}\right)$ for $p\left(\xi_{i}\right) \in$ $H_{*, *}\left[\xi_{1}, \cdots, \xi_{n}, \cdots\right]$ and $r\left(d_{j}\right) \in H_{*, *}\left[d_{i}, i \neq q^{n}-1, \forall n\right]$ where $\psi$ is the coproduct of $\mathcal{A}_{*, *}$. We set $\xi_{0}$ to be 1 .

Let us prove first that Theorem 4.3 implies Theorem 4.1. If $\theta \in \mathcal{A}_{m}^{* *}$ and $\sigma \in H^{*, *}(\mathbf{X}, \mathbb{Z} / q)$ for some spectrum $\mathbf{X}$, we will occasionally write $\theta^{\mathcal{A}} \cdot \sigma$ to denote the class in $H^{* * *}(\mathbf{X}, \mathbb{Z} / q)$ obtained by applying the cohomological operation $\theta$ to the cohomology class $\sigma$, when the simple notation $\theta \sigma$ may appear confusing. [2, Theorem 3] and the fact that $H^{u, v}(\operatorname{Spec} k, \mathbb{Z} / q)=0$ for $v>u$ implies that 
$Q_{i}{ }^{\mathcal{A}} \cdot c=0$ for any class in $c \in H^{2 *, *}(\mathbf{M G l}, \mathbb{Z} / q)$ for all $i \geq 0$ simply by degree considerations. Thus, the left $\mathcal{A}^{* *}$ action on the classes $d_{I}^{\prime}$ factors through $\frac{\mathcal{A}^{* *}}{\mathcal{A}^{* *}\left(Q_{0}, \cdots, Q_{t}, \cdots\right)}$. We now prove that $\frac{\mathcal{A}^{* *}}{\mathcal{A}^{* *}\left(Q_{0}, \cdots, Q_{t}, \cdots\right)} \cdot d_{I}^{\prime} \cong \frac{\mathcal{A}^{* *}}{\mathcal{A}^{* *}\left(Q_{0}, \cdots, Q_{t}, \cdots\right)}$. Recall that $\xi_{k}$ have the following $\mathcal{A}_{*, *}$ coproduct:

$$
\psi\left(\xi_{k}\right)=\sum_{i=0}^{k} \xi_{k-i}^{q^{i}} \otimes \xi_{i}
$$

(cf. [13] or [5]). Assume Theorem 4.3 holds true. The duality between $\mathcal{A}^{* *}$ action and $\mathcal{A}_{*, *}$ coaction means that $\langle\theta \stackrel{\mathcal{A}}{\cdot} y, x\rangle=\langle\theta \otimes y, \psi(x)\rangle$ for all $\theta \in \mathcal{A}^{* *}, y \in$ $H^{*, *}(\mathbf{M G l}, \mathbb{Z} / q)$ and $x \in H_{*, *}(\mathbf{M G l}, \mathbb{Z} / q)$. For any $I=\left(i_{1}, \cdots, i_{k}\right)$, we let $d_{I}=d_{1}^{i_{1}} \cdots d_{k}^{i_{k}}$ and $d_{I}^{\prime}$ be the Kronecker dual to $d_{I}$ and $1 \otimes d_{I}^{\prime} \in H^{*, *}(\mathbf{M G l}, \mathbb{Z} / q)$ the Kronecker dual to $1 \otimes d_{I} \in H_{*, *}(\mathbf{M G l}, \mathbb{Z} / q)$. Using the previous rules, we prove that, for $I$ fixed, the class $\left(r_{1}, \cdots, r_{n}\right)^{\mathcal{A}}\left(1 \otimes d_{I}^{\prime}\right)$ is always nonzero. Indeed,

$$
\begin{aligned}
& \left\langle\left(r_{1}, \cdots, r_{n}\right) \cdot \mathcal{A}\left(1 \otimes d_{I}^{\prime}\right), \xi_{1}^{r_{1}} \cdots \xi_{n}^{r_{n}} \otimes x\right\rangle \\
& =\left\langle\left(r_{1}, \cdots, r_{n}\right) \otimes\left(1 \otimes d_{I}^{\prime}\right), \prod_{k=1}^{n} \psi\left(\xi_{k}\right)^{r_{k}} \otimes x\right\rangle \\
& =\left\langle\left(r_{1}, \cdots, r_{n}\right) \otimes\left(1 \otimes d_{I}^{\prime}\right), \prod_{k=1}^{n}\left(\sum_{j=0}^{k} \xi_{k-j}^{q^{j}} \otimes \xi_{j}\right)^{r_{k}} \otimes x\right\rangle .
\end{aligned}
$$

By definition of $\left(r_{1}, \cdots, r_{n}\right)$ and $d_{I}^{\prime}$, this integer is nonzero and equals 1 only if $x=d_{I}$, since it equals to $\left\langle\left(r_{1}, \cdots, r_{n}\right), \xi_{1}^{r_{1}} \cdots \xi_{n}^{r_{n}}\right\rangle\left\langle 1 \otimes d_{I}^{\prime}, 1 \otimes d_{I}\right\rangle$. Because of the Remark 4.2, this proves Theorem 4.1.

Proof of Theorem 4.3. We will proceed by steps.

Step 1. Let $\gamma_{1}$ be the universal line bundle on $\mathbb{P}^{\infty} \cong \mathrm{Gr}_{1}$ so that we have $t$ : $H^{*, *}\left(\mathrm{Gr}_{1}, \mathbb{Z} / q\right) \stackrel{\cong}{\rightarrow} \widetilde{H}^{*+2, *+1}\left(\operatorname{Th}\left(\gamma_{1}\right), \mathbb{Z} / q\right)$, the Thom isomorphism. We wish to describe the left $\mathcal{A}^{* *}$ action on $\tilde{H}^{*, *}\left(\operatorname{Th}\left(\gamma_{1}\right), \mathbb{Z} / q\right)$. In $H^{*, *}\left(\mathbb{P}\left(\gamma_{1} \times \mathbb{A}_{k}^{1}\right), \mathbb{Z} / q\right)$ this homomorphism can be described as $-\cup c^{\operatorname{rank}\left(\gamma_{1}\right)}=-\cup c$, where $c \in H^{2,1}\left(\mathbb{P}\left(\gamma_{1} \times\right.\right.$ $\left.\left.\mathbb{A}_{k}^{1}\right), \mathbb{Z} / q\right)$ is the pull back of the polynomial generator $x$ of $H^{*, *}\left(\mathbb{P}^{\infty}, \mathbb{Z} / q\right) \cong$ $H^{*, *}[x]$ via the map classifying the universal line bundle over $\mathbb{P}\left(\gamma_{1} \times \mathbb{A}_{k}^{1}\right)$. In our case, by the Projective Bundle theorem (cf. [2]) and definition of Chern classes $c_{i}$, the powers of $c$ are subject to the relation

$$
c^{2}=c_{1}\left(\gamma_{1} \times \mathbb{A}_{k}^{1}\right) c-c_{2}\left(\gamma_{1} \times \mathbb{A}_{k}^{1}\right)
$$

which simplifies to $c^{2}=x c$, where

$$
H^{*, *}\left(\mathrm{Gr}_{1}, \mathbb{Z} / q\right)=H^{*, *}\left(\mathbb{P}^{\infty}, \mathbb{Z} / q\right) \cong H^{*, *}[x]
$$


and $x=c_{1}\left(\gamma_{1}\right)$. In other words, ${ }^{1}$

$$
H^{*, *}\left(\mathbb{P}\left(\gamma_{1} \times \mathbb{A}^{1}\right), \mathbb{Z} / q\right) \cong H^{*, *}[c, x] /\left(c^{2}-x c\right) .
$$

The canonical map $p: \mathbb{P}\left(\gamma_{1} \times \mathbb{A}_{k}^{1}\right) \rightarrow \operatorname{Th}\left(\gamma_{1}\right)$, in motivic cohomology, identifies isomorphically the group $\widetilde{H}^{*+2, *+1}\left(\mathrm{Th}\left(\gamma_{1}\right), \mathbb{Z} / q\right)$ with the subgroup $H^{* * *}\left(\mathrm{Gr}_{1}, \mathbb{Z} / q\right)$. $c$ of $H^{* * *}\left(\mathbb{P}\left(\gamma_{1} \times \mathbb{A}_{k}^{1}\right), \mathbb{Z} / q\right)$. Define $y_{i}$ as

$$
\widetilde{H}^{2 i, i}\left(\mathrm{Th}\left(\gamma_{1}\right), \mathbb{Z} / q\right) \ni y_{i}:=\left(p^{*}\right)^{-1}\left(x^{i-1} c\right)
$$

for $i>0$. By the Thom isomorphism, $\widetilde{H}^{*, *}\left(\operatorname{Th}\left(\gamma_{1}\right), \mathbb{Z} / q\right) \cong \oplus_{i} H^{*, *} y_{i}$ as left $H^{*, *}$ module and $\widetilde{H}_{*, *}\left(\operatorname{Th}\left(\gamma_{1}\right), \mathbb{Z} / q\right) \cong \oplus_{i} H_{*, *} y_{i}^{\prime}$, where $y_{i}^{\prime}$ are the classes dual to $y_{i}$. We want to understand the $\mathcal{A}_{*, *}$ coaction $\psi\left(y_{i}^{\prime}\right)$ of $y_{i}^{\prime} \in \widetilde{H}_{*, *}\left(\operatorname{Th}\left(\gamma_{1}\right), \mathbb{Z} / q\right)$ by means of the $\mathcal{A}^{* *}$ action on $y_{i}$. By the very definition of $y_{i}$ (equation (4.7)), and the Cartan formulae ([13, Proposition 9.6]) this reduces to the $\mathcal{A}^{* *}$ action on $x \in H^{2,1}\left(\mathbb{P}^{\infty}, \mathbb{Z} / q\right)$ and on $c$. Because of [13, Lemma 9.7] and Cartan formulae, we see that if $q=2, \theta^{\mathcal{A}} \cdot x \neq 0$ if and only if $\theta=a S q^{2^{n-1}} S q^{2^{n-2}} \cdots S q^{4} S q^{2}$ for some $a \in H^{* * *}(\operatorname{Spec} k, \mathbb{Z} / q)$ and $n$ positive integer and in that case $\theta^{\mathcal{A}}$. $x=a x^{2^{n}}$. If $q$ is odd then we conclude similarly that $\theta$ must be of the kind $a P^{q^{n-1}} P^{q^{n-2}} \ldots P^{q} P^{1}$ and the result is $a x^{q^{n}}$. If $i=1$ and $q=2, \theta^{\mathcal{A}} \cdot y_{1} \neq 0$ if and only if $\theta=a S q^{2^{n-1}} \cdots S q^{4} S q^{2}$ (and $\theta y_{1}=a y_{2^{n}}$ ), whereas if $q$ is odd, $\theta=a P^{q^{n-1}} P^{q^{n-2}} \ldots P^{q} P^{1}$ (and $\theta y_{1}=a y_{q^{n}}$ ). By employing the equality $y_{i}=$ $\left(p^{*}\right)^{-1}\left(x^{i-1} c\right)$ and the relation $c^{2}=x c$ we see that: $\theta^{\mathcal{A}} \cdot y_{n}=\sum_{i} a_{i} y_{i}$ if and only if $\theta^{\mathcal{A}} \cdot x^{n}=\sum_{i} a_{i} x^{i}$.

Step 2. We need a better understanding of the $\mathcal{A}_{*, *}$ coaction on $\tilde{H}_{*, *}\left(\operatorname{Th}\left(\gamma_{1}\right), \mathbb{Z} / q\right)$ :

Lemma 4.4. Let $\psi: \tilde{H}_{*, *}\left(\operatorname{Th}\left(\gamma_{1}\right), \mathbb{Z} / q\right) \rightarrow \mathcal{A}_{*, *} \otimes_{H_{*, *}} \tilde{H}_{*, *}\left(\operatorname{Th}\left(\gamma_{1}\right), \mathbb{Z} / q\right)$ the $\mathcal{A}_{*, *}$ coaction. Then for any prime number $q$

$$
\operatorname{Im}(\psi) \subset H_{*, *}\left[\xi_{1}, \xi_{2}, \cdots, \xi_{n}, \cdots\right] \otimes_{H_{*, *}} \tilde{H}_{*, *}\left(\operatorname{Th}\left(\gamma_{1}\right), \mathbb{Z} / q\right) .
$$

Proof. Since $\psi$ is $H_{*, *}$ linear, it suffices to consider $\psi\left(y_{i}^{\prime}\right)$ for all $i$. We recall that $a_{i}-2 b_{i}=1$ if $\left|Q_{i}\right|=\left(a_{i}, b_{i}\right)$. This implies that $Q_{i} \stackrel{\mathcal{A}}{\cdot} x=0$ and consequently $Q_{i} \stackrel{\mathcal{A}}{\cdot} y_{1}=0$ for all $i \geq 0$. In view of the duality between the action of $\mathcal{A}^{* *}$ and the coaction of the dual and motivic cohomology of $\operatorname{Th}\left(\gamma_{1}\right)$ and the fact that $Q_{i}\left(r_{1}, \cdots, r_{n}\right)$ is dual to $\tau_{i} \xi_{1}^{r_{1}} \cdots \xi_{n}^{r_{n}}$, to prove the lemma it suffices to show that $Q_{n} \stackrel{\mathcal{A}}{\cdot} y_{i}=0$ for all $n$ and $i$. As in Step $1 . Q_{n} \stackrel{\mathcal{A}}{\cdot} y_{i}$ can be computed by means of

${ }^{1}$ Probably $x=c$, but we will not need this fact in the computations. 
$Q_{n}{ }^{\mathcal{A}} \cdot x^{i}$. More precisely, $Q_{n}{ }^{\mathcal{A}} \cdot x^{i}=\sum_{j=0}^{i-1} x^{j} Q_{n}{ }^{\mathcal{A}} \cdot x x^{i-j-1}+\sum \rho^{h} p(x)$, where $p(x)$ are polynomials whose monomials contain at least a factor of the kind $Q_{s} \cdot{ }^{\mathcal{A}} x$ ( $c f$. [2, Corollary 5]), therefore the whole expression is zero. By the last remark in Step 1 this implies $Q_{n} \stackrel{\mathcal{A}}{\cdot} y_{i}=0$.

Step 3. Now we will exibit a different $H_{*, *}$ polynomial algebra basis for $H_{*, *}\left(\mathrm{Gr}_{\mathrm{n}}, \mathbb{Z} / q\right)$ than the one described in [2], which will be more useful in our argument. Let $\mu: \prod_{i=1}^{n} \mathrm{Gr}_{1} \rightarrow \mathrm{Gr}_{\mathrm{n}}$ classifying $\prod_{i=1}^{n} \gamma_{1, i}$. $\mu$ can be employed in the usual way to obtain a map, which we will call $\mu_{*}: \otimes_{i=1}^{n} H_{*, *}\left(\mathrm{Gr}_{1}, \mathbb{Z} / q\right) \rightarrow$ $H_{*, *}\left(\mathrm{Gr}_{\mathrm{n}}, \mathbb{Z} / q\right)$. In what follows we set $z_{k_{i}} \in H_{2 k_{i}, k_{i}}\left(\mathrm{Gr}_{1}, \mathbb{Z} / q\right)$ to be the Kronecker dual to $c_{1}^{k_{i}}\left(\gamma_{1, i}\right)$, that is the $k_{i}$-th power of the first Chern class of the universal line bundle on the $i$-th copy of $\mathrm{Gr}_{1}$ in $\mu: \prod_{i=1}^{n} \mathrm{Gr}_{1} \rightarrow \mathrm{Gr}_{\mathrm{n}}$. We wish to show that all the elements $b_{k_{1}} \cdots b_{k_{m}}:=\mu_{*}\left(z_{k_{1}} \otimes \cdots \otimes z_{k_{m}}\right)$ form an $H_{*, *}$ basis of $H_{*, *}\left(\mathrm{Gr}_{\mathrm{n}}, \mathbb{Z} / q\right)$ for $k_{1} \geq \cdots \geq k_{m} \geq 0$ and $m \leq n$. To do this, notice that we can order lexicographically from right to left such $b_{I}\left(e . g\right.$. $\left.b_{6} b_{5} b_{0}<b_{2} b_{1} b_{1}\right)$ so that it suffices to give, for each monomial in the Chern classes $f\left(c_{i}\right)=c_{1}^{e_{1}}, \cdots c_{n}^{e_{n}}$, a class $b_{f}$ with the properties that $\left\langle f\left(c_{i}\right), b_{f}\right\rangle=1,\left\langle f\left(c_{i}\right), b_{I}\right\rangle=0$ for $b_{I}<b_{f}$ and $b_{f} \neq b_{f^{\prime}}$ if $f \neq f^{\prime}$. Because we know that the Chern classes are polynomial generators of the motivic cohomology of $\mathrm{Gr}_{\mathrm{n}}$, this proves the claim. Given two vector bundles $v$ and $\eta$ over $X$ we have that $c_{h}(v \oplus \eta)=\sum_{i=0}^{h} c_{i}(v) \cup c_{h-i}(\eta)$ from which it follows that $c_{h}(\nu \times \eta)=\sum_{i=0}^{h} c_{i}(\nu) \times c_{h-i}(\eta) \in H^{* * *}(X \times \operatorname{Spec} k X, \mathbb{Z} / q)$. This shows that $c_{h}\left(\prod_{i=1}^{n} \gamma_{1, i}\right)=\sum_{j_{1}<\cdots<j_{h}} x_{j_{1}} \otimes \cdots \otimes x_{j_{h}}$, that is the elementary $h$-th symmetric polynomial in the variables $x_{i}:=c_{1}\left(\gamma_{1, i}\right)$. We have the chain of equalities

$$
\begin{aligned}
\left\langle c_{1}^{e_{1}} \cdots c_{n}^{e_{n}}, \mu_{*}\left(z_{k_{1}} \otimes \cdots \otimes z_{k_{n}}\right)\right\rangle & =\left\langle\mu^{*}\left(c_{1}^{e_{1}} \cdots c_{n}^{e_{n}}\right), z_{k_{1}} \otimes \cdots \otimes z_{k_{n}}\right\rangle \\
& =\left\langle\mu^{*}\left(c_{1}\right)^{e_{1}} \cdots \mu^{*}\left(c_{n}\right)^{e_{n}}, z_{k_{1}} \cdots z_{k_{n}}\right\rangle
\end{aligned}
$$

but $\mu^{*}\left(c_{h}\right)=c_{h}\left(\prod_{i=1}^{n} \gamma_{1, i}\right)$ and the smallest, according to the order given above, homology class $b_{I}$ which has nonzero (actually equals to 1 ) Kronecker pairing with it comes from $z_{1} \otimes \stackrel{h}{ } \otimes \otimes z_{1} \otimes z_{0} \cdots \otimes z_{0}$ where we let $z_{0}=1$. Similarly we see that the smallest class with nonzero (and equals to 1 ) Kronecker pairing with $c_{1}^{e_{1}} \cdots c_{n}^{e_{n}}$ is

$$
b_{e_{1}+\cdots+e_{n}} b_{e_{2}+\cdots+e_{n}} \cdots b_{e_{n-1}+e_{n}} b_{e_{n}} .
$$

This is the class we denoted $b_{f}$ above.

Step 4. (end of the proof of Theorem 4.3) We donote by $t_{i}$ the homology Thom isomorphisms on the homology of $\mathrm{Gr}_{i}$. By the commutativity of the diagram (which is consequence of the naturality of the Thom isomorphism) 


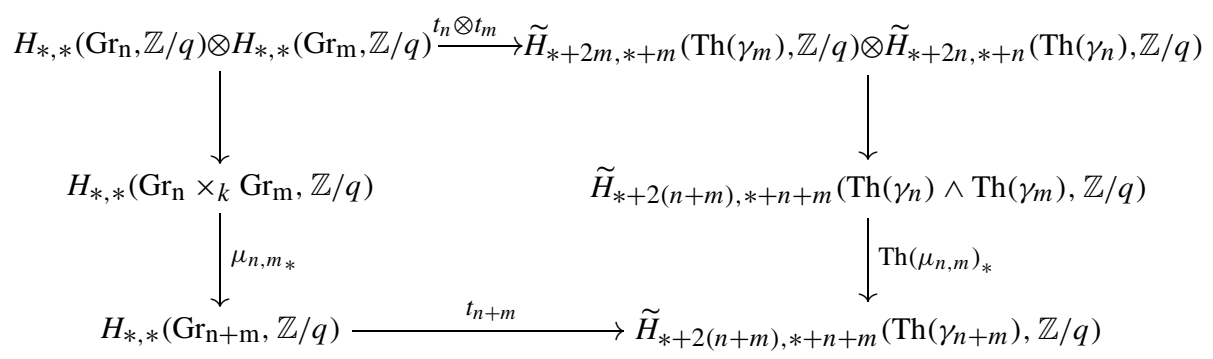

we see that the equality in $H_{*, *}(\mathbf{M G l}, \mathbb{Z} / q)$

$$
t_{n}\left(b_{k_{1}} \cdot b_{k_{s}}\right) \cdots t_{m}\left(b_{h_{1}} \cdots b_{h_{r}}\right)=t_{n+m}\left(b_{k_{1}} \cdots b_{k_{s}} b_{h_{1}} \cdots b_{h_{r}}\right)
$$

holds, thus $t_{h}\left(b_{k_{1}} \cdots b_{k_{v}}\right)=t_{1}\left(b_{k_{1}}\right) \cdots t_{1}\left(b_{k_{v}}\right)$. The ring structure in the motivic homology of both Gr and MGl is the Pontryagin product. We will take the monomials $t_{1}\left(b_{k_{1}}\right) \cdots t_{1}\left(b_{k_{l}}\right)$ for all $l>0$ as left $H_{*, *}$ basis for $\widetilde{H}_{*, *}(\mathbf{M G l}, \mathbb{Z} / q)$. By naturality of the $\mathcal{A}_{*, *}$ coaction $\psi$ and because of Lemma 4.4, we conclude that

$$
\psi\left(\tilde{H}_{*, *}(\mathbf{M G l}, \mathbb{Z} / q)\right) \subset H_{*, *}\left[\xi_{1}, \cdots \xi_{n}, \cdots\right] \otimes_{H_{*, *}} \tilde{H}_{*, *}(\mathbf{M G l}, \mathbb{Z} / q) .
$$

Consider the composition

$$
\begin{aligned}
& \lambda: \tilde{H}_{*, *}(\mathbf{M G l}, \mathbb{Z} / q) \stackrel{\psi}{\rightarrow} H_{*, *}\left[\xi_{1}, \cdots \xi_{n}, \cdots\right] \otimes_{H_{*, *}} \tilde{H}_{*, *}(\mathbf{M G l}, \mathbb{Z} / q) \stackrel{1 \otimes f}{\rightarrow} \\
& \rightarrow H_{*, *}\left[\xi_{1}, \cdots \xi_{n}, \cdots\right] \otimes_{H_{*, *}} H_{*, *}\left[d_{i}, i \neq q^{n}-1, \forall n\right]
\end{aligned}
$$

the map $f$ being the ring homomorphism defined by $f\left(t_{1}(1)\right)=1=d_{0}, f\left(t_{1}\left(b_{i}\right)\right)=$ $d_{i}$ if $i \neq q^{k}-1$ for any $k$ and $f\left(t_{1}\left(b_{q^{k}-1}\right)\right)=0$.

Since both the domain and the target are polynomial algebras with one generator in bidegree $(2 i, i)$ for all $i>0$. To prove that $\lambda$ is an isomorphism, it suffices to show that the image through this map of each polynomial generator is indecomposable. In Step 1 we have shown that the Thom isomorphism in motivic cohomology sends $x^{i} \in H^{2 i, i}\left(\mathrm{Gr}_{1}, \mathbb{Z} / q\right)=H^{2 i, i}\left(\mathbb{P}^{\infty}, \mathbb{Z} / q\right)$ to $y_{i+1} \in \tilde{H}^{2(i+1), i+1}\left(\operatorname{Th}\left(\gamma_{1}\right), \mathbb{Z} / q\right)$, thus letting $b_{i}$ to be the dual to $x^{i}, t_{1}\left(b_{i}\right)=y_{i+1}^{\prime}$. If $i=q^{k}-1, \psi\left(t_{1}\left(b_{i}\right)\right)=$ $\psi\left(y_{q^{k}}^{\prime}\right)=1 \otimes y_{q^{t}}^{\prime}+\xi_{k} \otimes y_{1}^{\prime}+\cdots$ and since $y_{1}^{\prime}=t_{1}(1)$, we have that $\lambda\left(t_{1}\left(b_{i}\right)\right)=$ $\xi_{k} \otimes 1+\cdots$ hence it is indecomposable. If $i \neq q^{k}-1, \psi\left(t_{1}\left(b_{i}\right)\right)=1 \otimes t_{1}\left(b_{i}\right)+\cdots$ and $\lambda\left(t_{1}\left(b_{i}\right)\right)=1 \otimes d_{i}+\cdots$ which is still indecomposable. Thinking of $\lambda$ as a morphism in the category of $\mathcal{A}_{*, *}$ comodule algebras by endowing the tensor products of the $\psi \otimes i d$ coaction, we conclude the last part of Theorem 4.3.

At this point the construction proceeds as in [2]. Thanks to the previous theorem we can kill the homotopy classes described in Theorem 3.1 and keep track of the changements that occur in motivic cohomology when we pass from $\mathbf{E}_{i-1}$ to $\mathbf{E}_{i}$ for each $i$ of the diagram (4.1). For the explicit calculations, we refer to the corresponding results in the proof of [2, Theorem 12]. They can be employed in our present context without any changements. 
Definition 4.5. Denote $k^{\prime}(t)$ the spectrum

$$
\operatorname{hocolim}_{i}\left\{\mathbf{M G l} \stackrel{\eta_{0}}{\rightarrow} \mathbf{E}_{0} \stackrel{\eta_{1}}{\rightarrow} \mathbf{E}_{1} \cdots \rightarrow \mathbf{E}_{n} \stackrel{\eta_{n+1}}{\rightarrow} \cdots\right\}
$$

Let $\mathcal{A}^{* *}$ be the left $H^{*, *}$ submodule of $\left[\mathbf{H}_{\mathbb{Z} / q}, \Sigma^{i, j} \mathbf{H}_{\mathbb{Z} / q}\right]_{\mathcal{S H}(k)}$ generated by monomials in $S q^{i}$, if $q=2$ and by $P^{i}$ and the Bockstein $\beta$, if $q$ is odd (see $[2,13]$ ).

Theorem 4.6. The spectra $k^{\prime}(t)$, as defined above, have the property that

$$
H^{*, *}\left(k^{\prime}(t), \mathbb{Z} / q\right) \cong \frac{\mathcal{A}^{* *}}{\mathcal{A}^{* *} Q_{t}} \tau \cong \frac{\mathcal{A}^{* *}}{\mathcal{A}^{* *} Q_{t}}
$$

as left $\mathcal{A}^{* *}$ module and $\tau \in H^{0,0}\left(k^{\prime}(t), \mathbb{Z} / q\right)$ is the canonical class corresponding to the Thom class in $H^{0,0}(\mathbf{M G l}, \mathbb{Z} / q)$ via the canonical morphism MGl $\rightarrow k^{\prime}(t)$. Moreover, there exists an exact triangle $\Sigma^{2\left(q^{t}-1\right), q^{t}-1} k^{\prime}(t) \stackrel{v_{t}}{\rightarrow} k^{\prime}(t) \rightarrow C_{0}$, with $H^{*, *}\left(C_{0}, \mathbb{Z} / q\right) \cong \mathcal{A}^{* *} \iota^{\prime}, \iota^{\prime} \in H^{0,0}\left(C_{0}, \mathbb{Z} / q\right)$ being the class corrsponding to $\tau$.

Proof. See [2, Theorem 12].

Let $\mathcal{B}^{* *}$ be the kernel of $\left(\iota^{\prime}\right)^{*}$ where $\iota^{\prime}: C_{0} \rightarrow \mathbf{H}_{\mathbb{Z} / q}$ is the class as in the statement of the theorem. Then, as left $\mathcal{A}^{* *}$ module,

$$
\left[\mathbf{H}_{\mathbb{Z} / q}, \Sigma^{*, *} \mathbf{H}_{\mathbb{Z} / q}\right]_{\mathcal{S H}(k)}=: \mathcal{A}_{m}^{* *} \cong \mathcal{A}^{* *} \oplus \mathcal{B}^{* *}
$$

Corollary 4.7. $b \in \mathcal{B}^{* *}$ if and only if $b^{\mathcal{A}} \cdot \iota^{\prime}=0$. Furthermore, if $b \in \mathcal{B}^{* *}$, $b^{\mathcal{A}} \cdot \tau=0$ and hence $\left(b Q_{t}\right) \stackrel{\mathcal{A}}{\cdot} \iota^{\prime}=b^{\mathcal{A}} \cdot\left(Q_{t} \cdot \mathcal{A} \cdot \iota^{\prime}\right)=0$ as well.

Proof. The first two statements follow from the definition of action of a cohomological operation on a class. The third is proven via the commutativity of the square

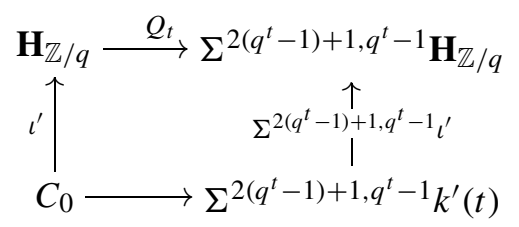

and the second statement that implies $b^{\mathcal{A}} \cdot \tau=0$.

\section{The spectra $\Phi_{i}$}

After the construction of $k^{\prime}(t)$, the reason for proceeding further, lies in the fact that we do not know if these spectra are built out of suspensions of motivic EilenbergMacLane spectra $\Sigma^{*, *} \mathbf{H}_{\mathbb{Z} / q}$. Such property is valid for the spectra $\boldsymbol{\Phi}_{i}$ described below and is needed to derive the degree formulae of [1], because we know that 
Eilenberg MacLane spectra represent the Chow groups functor under the assumption of perfectness of the base field. We will use what proved so far on the spectra $k^{\prime}(t)$ to prove the main result. The $\mathbb{Z} / q$ algebra $\mathcal{A}_{m}^{* *}$ has a left and right action by $\mathcal{A}^{* *}$ induced by the multiplicative product of $\mathcal{A}_{m}^{* *}$ and the canonical (split) inclusion $\mathcal{A}^{* *} \hookrightarrow \mathcal{A}_{m}^{* *}$ described at the end of Section 4. Recall that the Margolis homology $H M\left(M, Q_{t} \cdot\right)$ (resp. $H M\left(M, \cdot Q_{t}\right)$ ) of a left (resp. right) module $M$ over $\mathcal{A}^{* *}$ is defined as the first homology group of the complex $\left\{M \stackrel{Q_{t}}{\rightarrow} M \stackrel{Q_{t}}{\rightarrow} M\right\}$ (resp. of $\left.\left\{M \stackrel{Q_{t}}{\rightarrow} M \stackrel{Q_{t}}{\rightarrow} M\right\}\right)$. Let us denote by $P$ the following condition: if $a$ be a class in $\mathcal{B}^{* *} / \mathcal{B}^{* *} Q_{t}$ of degree $\left(2 j\left(q^{t}-1\right)+2(j-1), j\left(q^{t}-1\right)\right)$, then there exists a class $b \in \mathcal{B}^{* *} / \mathcal{B}^{* *} Q_{t}$ such that $a=Q_{t} b$, for $3 \leq j$, where $Q_{t}$ acts by left multiplication as an element of $\mathcal{A}_{m}^{* *}$. We are going to make one of the following assumptions on the left $\mathcal{A}^{* *}$ module $\mathcal{B}^{* *}$ :

(1) either $P$ and the same $Q_{t}$-divisibility property hold for all classes $a \in \mathcal{B}^{* *} / \mathcal{B}^{* *} Q_{t}$ of degree $\left(2(j-2)\left(q^{t}-1\right)+(j-2),(j-2)\left(q^{t}-1\right)\right)$,

(2) or $P$ and the same $Q_{t}$-divisibility property hold for all classes $a \in H M\left(\mathcal{B}^{* *}, Q_{t}\right)$ of degree $\left(2(j-2)\left(q^{t}-1\right)+(j-2),(j-2)\left(q^{t}-1\right)\right)$.

Theorem 5.1. Let $k$ be any perfect field and $q$ a fixed prime number. Suppose that $\mathcal{A}_{m}^{* *}$ satisfies the assumption listed above. Then, for each $1 \leq i \leq r-1$, there exists a spectrum $\boldsymbol{\Phi}_{i}$ in the stable category $\mathcal{S H}(k)$ fitting in exact triangles

$$
\boldsymbol{\Phi}_{i} \longrightarrow \boldsymbol{\Phi}_{i-1} \stackrel{y_{i-1}}{\longrightarrow} \Sigma^{2 i\left(q^{t}-1\right)+1, i\left(q^{t}-1\right)} \mathbf{H}_{\mathbb{Z} / q} .
$$

Let $\iota=1 \in H^{0,0}\left(\mathbf{H}_{\mathbb{Z} / q}, \mathbb{Z} / q\right)=\mathcal{A}_{m}^{* *}$ and

$$
\delta: H^{*, *}\left(\boldsymbol{\Phi}_{i}, \mathbb{Z} / q\right) \rightarrow H^{*+1, *}\left(\Sigma^{2 i\left(q^{t}-1\right), i\left(q^{t}-1\right)} \mathbf{H}_{\mathbb{Z} / q}, \mathbb{Z} / q\right)
$$

be the coboundary operator induced by the exact triangle defining $\boldsymbol{\Phi}_{i}$ in function of $y_{i-1}$ and $\boldsymbol{\Phi}_{i-1}$. Then the classes $y_{i}$ are subject to the relation $\delta y_{i}=$ $Q_{t} \Sigma^{2 i\left(q^{t}-1\right), i\left(q^{t}-1\right)} \iota$.

We mention that [2, Lemma 14] can be employed to derive another kind of exact triangle involving the spectra $\boldsymbol{\Phi}_{i}$ :

$$
\Sigma^{2\left(q^{t}-1\right), q^{t}-1} \boldsymbol{\Phi}_{i-1} \longrightarrow \boldsymbol{\Phi}_{i} \stackrel{\tau_{i}}{\longrightarrow} \mathbf{H}_{\mathbb{Z} / q} .
$$

Proof of Theorem 5.1. Let $d=q^{t}-1$. We first prove an algebraic result concerning certain left $\mathcal{A}^{* *}$ modules. More precisely, we will recursively solve an algebraic problem following steps which correspond to the index $i$ as in the spectrum $\boldsymbol{\Phi}_{i}$. Such algebraic picture is motivated by the homotopical approach to the construction of the $\boldsymbol{\Phi}_{i}$ as depicted in the diagram (5.15). At each step, we will use the assumptions (1) and (2) to prove the existence of a class $y_{i}^{\prime}$ satisfying few properties. Its existence allows us to proceed inductively to the next step. 
Step 0. Consider the short exact sequence of left $\mathcal{A}_{m}^{* *}$ modules

$$
0 \rightarrow \operatorname{ker}\left(p_{0}\right) \rightarrow \mathcal{A}_{m}^{* *} \stackrel{p_{0}}{\rightarrow} \frac{\mathcal{A}^{* *}}{\mathcal{A}^{* *} Q_{t}} \rightarrow 0
$$

where $p_{0}(1)=1$. Writing $\mathcal{A}_{m}^{* *} \cong \mathcal{A}^{* *} \oplus \mathcal{B}^{* *}$ as left $\mathcal{A}^{* *}$ modules and because of Corollary 4.7 , we conclude that $\operatorname{ker}\left(p_{0}\right) \cong \mathcal{A}^{* *} \cdot Q_{t} \oplus \mathcal{B}^{* *}$ as left $\mathcal{A}^{* *}$ modules. Since the $\cdot Q_{t}$ Margolis homology of $\mathcal{A}^{* *}$ vanishes [2, Theorem 9], $\operatorname{ker}\left(p_{0}\right) \cong\left(\frac{\mathcal{A}^{* *}}{\mathcal{A}^{* *} Q_{t}} \cdot y_{0}^{\prime}\right) \oplus \mathcal{B}^{* *}$, where we let $y_{0}^{\prime}$ to be $Q_{t}$.

Step 1. Consider now the new exact sequence of graded left $\mathcal{A}_{m}^{* *}$ modules

$$
0 \rightarrow \operatorname{ker}\left(p_{1}\right) \rightarrow \mathcal{A}_{m}^{* *} \Sigma^{2 d+1, d} \iota \stackrel{p_{1}}{\rightarrow} \operatorname{ker}\left(p_{0}\right) \cong \frac{\mathcal{A}^{* *}}{\mathcal{A}^{* *} Q_{t}} \cdot y_{0}^{\prime} \oplus \mathcal{B}^{* *} y_{0}^{\prime}
$$

where $p_{1}$ is defined by $p_{1}\left(\Sigma^{2 d+1, d} \iota\right)=y_{0}^{\prime}=Q_{t}$. Because of Corollary 4.7, multiplication to the right by $Q_{t}$ is an homomorphism both $\mathcal{A}^{* *} \rightarrow \mathcal{A}^{* *}$ and $\mathcal{B}^{* *} \rightarrow \mathcal{B}^{* *}$, thus we have

$$
\operatorname{ker}\left(p_{1}\right)=\frac{\mathcal{A}^{* *}}{\mathcal{A}^{* *} Q_{t}}\left(Q_{t} \Sigma^{2 d+1, d} \iota\right) \oplus \operatorname{ker}\left\{\mathcal{B}^{* *} \stackrel{Q_{t}}{\rightarrow} \mathcal{B}^{* *}\right\} \Sigma^{2 d+1, d} \iota
$$

as left $\mathcal{A}^{* *}$ modules. Later on, we are going to represent this exact sequence as part of a motivic cohomology long exact sequence between objects in $\mathcal{S H}(k)$. Thus, we wish to continue it on the left as if it were part of a long exact sequence of graded left $\mathcal{A}_{m}^{* *}$ modules:

$$
\cdots \stackrel{g_{1}}{\rightarrow} \mathcal{C}^{* *} \stackrel{f_{1}}{\rightarrow} \mathcal{A}_{m}^{* *} \Sigma^{2 d+1, d} \iota \stackrel{p_{1}}{\rightarrow} \frac{\mathcal{A}^{* *}}{\mathcal{A}^{* *} Q_{t}} \cdot y_{0}^{\prime} \oplus \mathcal{B}^{* *}
$$

where $\mathcal{C}^{* *}$ fits in a short exact sequence of graded left $\mathcal{A}_{m}^{* *}$ modules

$$
\begin{aligned}
0 & \rightarrow \Sigma^{-1,0}\left(\mathcal{B}^{* *} / \mathcal{B}^{* *} Q_{t}\right) \stackrel{g_{1}}{\rightarrow} \mathcal{C}^{* *} \stackrel{f_{1}}{\rightarrow} \\
& \rightarrow \frac{\mathcal{A}^{* *}}{\mathcal{A}^{* *} Q_{t}}\left(Q_{t} \Sigma^{2 d+1, d} \iota\right) \oplus \Sigma^{2 d+1, d} \operatorname{ker}_{\mathcal{B}^{* *}}\left(\cdot Q_{t}\right)=\operatorname{ker}\left(p_{1}\right) \rightarrow 0
\end{aligned}
$$

where we denoted $\operatorname{ker}\left\{\mathcal{B}^{* *} \stackrel{Q_{t}}{\rightarrow} \mathcal{B}^{* *}\right\}$ by $\operatorname{ker}_{\mathcal{B}^{* *}}\left(\cdot Q_{t}\right)$. Let $y_{1}^{\prime}$ be a lift of $Q_{t} \Sigma^{2 d+1, d} \iota$ to $\mathcal{C}^{* *}$. Composing $f_{1}$ with the projection $\operatorname{ker}\left(p_{1}\right) \rightarrow \operatorname{ker}\left(p_{1}\right) / \mathcal{A}_{m}^{* *}\left(Q_{t} \Sigma^{2 d+1, d} \iota\right)$, we see that $\mathcal{C}^{* *}$ fits in the exact sequence of graded left $\mathcal{A}_{m}^{* *}$ modules

$$
0 \rightarrow \Sigma^{-1,0}\left(\mathcal{B}^{* *} / \mathcal{B}^{* *} Q_{t}\right)+\mathcal{A}_{m}^{* *} y_{1}^{\prime} \rightarrow \mathcal{C}^{* *} \rightarrow \Sigma^{2 d+1, d} \operatorname{ker}_{\mathcal{B}^{* *}}\left(\cdot Q_{t}\right) / M \rightarrow 0
$$

Since $\cdot Q_{t}$ respects the splitting $\mathcal{A}_{m}^{* *}=\mathcal{A}^{* *} \oplus \mathcal{B}^{* *}$, we get that $M=\mathcal{B}^{* *} Q_{t}$. We wish to get a better understanding of the module $\mathcal{A}_{m}^{* *} y_{1}^{\prime}$. Since $\mathcal{A}_{m}^{* *} \cong \mathcal{A}^{* *} \oplus \mathcal{B}^{* *}$ 
we conclude that $\mathcal{A}_{m}^{* *} y_{1}^{\prime} \cong \mathcal{A}^{* *} y_{1}^{\prime}+\mathcal{B}^{* *} y_{1}^{\prime}$. Now, suppose that the property (5) holds. Then we have that $Q_{t} y_{1}^{\prime}=g_{1}\left(Q_{t} z\right)$ for some $z \in\left(\mathcal{B}^{* *} / \mathcal{B}^{* *} Q_{t}\right)^{4 d+3,2 d}$. But $g_{1}\left(Q_{t} z\right)=Q_{t} g_{1}(z)$ and letting $\hat{y}_{1}:=y_{1}^{\prime}-g_{1}(z)$ we see that $Q_{t} \hat{y}_{1}=0$. Summing up, the property (5) implies that we can choose $y_{1}^{\prime}$ in such a way that $Q_{t} y_{1}^{\prime}=0$. Suppose now that $a y_{1}^{\prime}=b y_{1}^{\prime}$ for $a \in \mathcal{A}^{* *}$ and $b \in \mathcal{B}^{* *}$, by applying $p_{1}$ we see that $a Q_{t}=b Q_{t}=0$ because right multiplication by $Q_{t}$ preserve the splitting of $\mathcal{A}_{m}^{* *}$. Since $H M\left(\mathcal{A}^{* *}, Q_{t}\right)=0$ we have that $a=a^{\prime} Q_{t}$, whence $a y_{1}^{\prime}=0$ by our assumption. This shows that

$$
\mathcal{A}_{m}^{* *} y_{1}^{\prime} \cong \frac{\mathcal{A}^{* *}}{\mathcal{A}^{* *} Q_{t}} y_{1}^{\prime} \oplus \mathcal{B}^{* *} y_{1}^{\prime}
$$

Using these new information, the sequence (5.7) becomes

$$
\begin{aligned}
0 & \rightarrow \Sigma^{-1,0} \frac{\mathcal{B}^{* *}}{\mathcal{B}^{* *} Q_{t}}+\left(\frac{\mathcal{A}^{* *}}{\mathcal{A}^{* *} Q_{t}} y_{1}^{\prime} \oplus \mathcal{B}^{* *} y_{1}^{\prime}\right) \\
& \rightarrow \mathcal{C}^{* *} \rightarrow \Sigma^{2 d+1, d} \operatorname{ker}_{\mathcal{B}^{* *}}\left(\cdot Q_{t}\right) / \mathcal{B}^{* *} Q_{t}=\Sigma^{2 d+1, d} H M\left(\mathcal{B}^{* *}, Q_{t}\right) \rightarrow 0 .
\end{aligned}
$$

We will use the description of the module $\mathcal{A}_{m}^{* *} y_{1}^{\prime}$ on the next step.

Step 2. The next short exact sequence is

$$
0 \rightarrow \operatorname{ker}\left(p_{2}\right) \rightarrow \mathcal{A}_{m}^{* *} \Sigma^{4 d+2,2 d} \iota \stackrel{p_{2}}{\rightarrow} \mathcal{C}^{* *}
$$

with $p_{2}\left(\Sigma^{4 d+2,2 d} \iota\right)=y_{1}^{\prime}$. We extend it to a long exact sequence

$$
\rightarrow \mathcal{D}^{* *} \stackrel{f_{2}}{\rightarrow} \mathcal{A}_{m}^{* *} \Sigma^{4 d+2,2 d} \stackrel{p_{2}}{\rightarrow} \mathcal{C}^{* *} .
$$

The following exact sequences give a better understanding of $\mathcal{D}^{* *}$.

(1) $\mathcal{D}^{* *}$ fits in the short exact sequence of graded left $\mathcal{A}^{* *}$ modules

$$
0 \rightarrow \Sigma^{-1,0} \operatorname{coker}\left(p_{2}\right) \rightarrow \mathcal{D}^{* *} \stackrel{f_{2}}{\rightarrow} \operatorname{ker}\left(p_{2}\right) \rightarrow 0
$$

(2) $\operatorname{coker}\left(p_{2}\right)$ fits in the short exact sequence

$$
0 \rightarrow \Sigma^{-1,0} \frac{\frac{\mathcal{B}^{* *}}{\mathcal{B}^{* *} Q_{t}}}{M_{1}} \rightarrow \operatorname{coker}\left(p_{2}\right) \rightarrow H M\left(\mathcal{B}^{* *}, \cdot Q_{t}\right) \Sigma^{2 d+1, d} \iota \rightarrow 0
$$

for some module $M_{1}$; 
(3) $\operatorname{ker}\left(p_{2}\right) \cong \mathcal{A}^{* *} Q_{t} \Sigma^{4 d+2,2 d} \iota \oplus \operatorname{Ann}_{\mathcal{B}^{* *}}\left(\cdot y_{1}^{\prime}\right) \Sigma^{4 d+2,2 d} \iota$ where the annihilator $\operatorname{Ann}_{\mathcal{B}^{* *}}\left(\cdot y_{1}^{\prime}\right)$ fits in $\mathcal{B}^{* *} Q_{t} \subset \operatorname{Ann}_{\mathcal{B}^{* *}}\left(\cdot y_{1}^{\prime}\right) \subset \operatorname{ker}_{\mathcal{B}^{* *}}\left(\cdot Q_{t}\right)$, by definition of $y_{1}^{\prime}$. To prove this last isomorphism we use the computation of $\mathcal{A}_{m}^{* *} y_{1}^{\prime}$ done in the previous step.

We use this identification of $\operatorname{ker}\left(p_{2}\right)$ to define the class $y_{2}^{\prime}$ as a lifting of $Q_{t} \Sigma^{4 d+2,2 d} \iota$ to $\mathcal{D}^{* *}$. For this we must have known that $Q_{t} \Sigma^{4 d+2,2 d} \iota$ belongs to $\operatorname{ker}\left(p_{2}\right)$. We conclude that $\mathcal{D}^{* *}$ fits in the short exact sequence

$$
0 \rightarrow \Sigma^{-1,0} \operatorname{coker}\left(p_{2}\right)+\mathcal{A}_{m}^{* *} y_{2}^{\prime} \rightarrow \mathcal{D}^{* *} \stackrel{f_{2}}{\rightarrow} \frac{\operatorname{Ann}_{\mathcal{B}^{* *}}\left(\cdot y_{1}^{\prime}\right)}{\mathcal{B}^{* *} Q_{t}} \Sigma^{4 d+2,2 d} \iota \rightarrow 0 .
$$

Notice that the previous observation, $\operatorname{Ann}_{\mathcal{B}^{* *}}\left(\cdot y_{1}^{\prime}\right) /\left(\mathcal{B}^{* *} Q_{t}\right) \subset H M\left(\mathcal{B}^{* *}, \cdot Q_{t}\right)$. As before, to set things for the next step and eventually define a $y_{3}^{\prime}$ we need more information on the module $\mathcal{A}_{m}^{* *} y_{2}^{\prime}$. We therefore use our assumption on $\mathcal{B}^{* *}$ to show the existence of a lifting $y_{2}^{\prime}$ such that $Q_{t} y_{2}^{\prime}=0$. To do this we must ensure that $\Sigma^{-1,0} \operatorname{coker}\left(p_{2}\right)$ is divisible by $Q_{t} \cdot$. By the exact sequence (3.1) we see that it suffices to assume that $\left(\mathcal{B}^{* *} / \mathcal{B}^{* *} Q_{t}\right)^{8 d+6,4 d}$ and $H M\left(\mathcal{B}^{* *}, Q_{t}\right)^{6 d+3,3 d}$ are divisible by $Q_{t}$ or $\left(\mathcal{B}^{* *} / \mathcal{B}^{* *} Q_{t}\right)^{8 d+6,4 d}$ and $\left(\mathcal{B}^{* *} / \mathcal{B}^{* *} Q_{t}\right)^{6 d+3,3 d}$ are divisible by $\cdot Q_{t}$.

Step n. Having a class $y_{n-1}^{\prime} \in \mathcal{D}_{n-1}^{* *}$ such that $Q_{t} y_{n-1}^{\prime}=0$ and $f_{n-1}\left(y_{n-1}^{\prime}\right)=$ $Q_{t} \Sigma^{2(n-1) d+(n-1), n-1} \iota$, we define $\mathcal{D}_{n}^{* *}$ as a module fitting in the long exact sequence

$$
\rightarrow \mathcal{D}_{n}^{* *} \rightarrow \mathcal{A}_{m}^{* *} \Sigma^{2 n d+n, n d} \stackrel{p_{n}}{\rightarrow} \mathcal{D}_{n-1}^{* *} \rightarrow \cdots
$$

It follows that $\mathcal{D}_{n}^{* *}$ lies in the short exact sequence

$$
0 \rightarrow \Sigma^{-n+1,0} \operatorname{coker}\left(p_{n}\right) \rightarrow \mathcal{D}_{n}^{* *} \rightarrow \operatorname{ker}\left(p_{n}\right) \rightarrow 0
$$

the structure of $\operatorname{ker}\left(p_{n}\right)$ is relevant to the existence of $y_{n}^{\prime}$, whereas $Q_{t}$. divisibility of $\operatorname{coker}\left(p_{n}\right)$ and of $\operatorname{ker}\left(p_{n-1}\right)$ are relevant to the existence of a $y_{n}^{\prime}$ with the property that $Q_{t} y_{n}^{\prime}=0$. We have that $\operatorname{ker}\left(p_{n}\right) \cong \mathcal{A}^{* *} Q_{t} \Sigma^{2 n d+n, n} \iota \oplus \operatorname{Ann}_{\mathcal{B}^{* *}}\left(\cdot y_{n-1}\right) \Sigma^{2 n d+n, n} \iota$, thus we can define $y_{n}^{\prime}$ as a lifting to $\mathcal{D}_{n}^{* *}$ of $Q_{t} \Sigma^{2 n d+n, n} \iota$. Finally coker $\left(p_{n}\right)$ is a multiple extension of suspended quotients of $\mathcal{B}^{* *} / \mathcal{B}^{* *} Q_{t}$ and of $H M\left(\mathcal{B}^{* *}, \cdot Q_{t}\right)$ hence of $\mathcal{B}^{* *} / \mathcal{B}^{* *} Q_{t}$ (the latter statement follows from the remark that $\operatorname{Ann}_{\mathcal{B}^{* *}}\left(\cdot y_{n-1}^{\prime}\right) /$ $\left.\left(\mathcal{B}^{* *} Q_{t}\right) \subset H M\left(\mathcal{B}^{* *}, Q_{t}\right) \subset \mathcal{B}^{* *} / \mathcal{B}^{* *} Q_{t}\right)$. The assumptions on the module $\mathcal{B}^{* *}$ set before the Theorem 5.1 imply the divisibility by $Q_{t}$. of $\Sigma^{-n+1,0} \operatorname{coker}\left(p_{n}\right)$ in degree $(2(n+2)+n+2, n+2)$, hence the existence of a choice of $y_{n}^{\prime}$ such that $Q_{t} y_{n}^{\prime}=0$.

We now perform constructions in the category $\mathcal{S H}(k)$ that will induce the exact sequences (5.3), (5.5), (5.11), .. up to some degree shifting. The classes $y_{i}^{\prime}$ will be used to the define the $y_{i}$ appearing in the statement of Theorem 5.1. Consider the 
following commutative diagram:

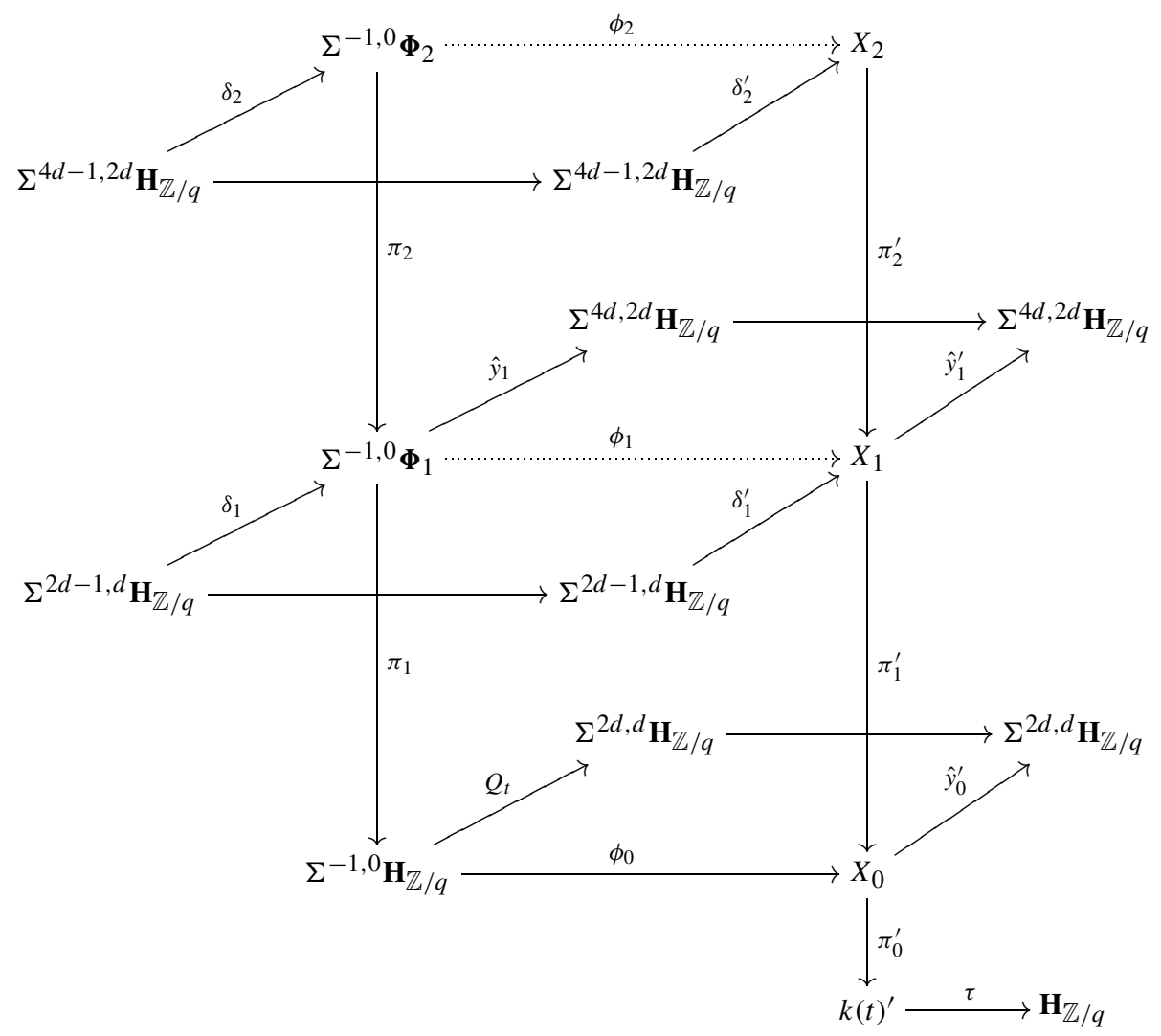

The triples $\left(\hat{y}_{i-1}, \pi_{i}, \delta_{i}\right)$ and $\left(\hat{y}_{i-1}^{\prime}, \pi_{i}^{\prime}, \delta_{i}^{\prime}\right)$ form exact triangles as well as $\left(\tau, \pi_{0}^{\prime}, \phi_{0}\right)$. The exact triangle $\left(\tau, \pi_{0}^{\prime}, \phi_{0}\right)$ induces the sequence (5.3), the triangle $\left(\hat{y}_{0}^{\prime}, \pi_{1}^{\prime}, \delta_{1}^{\prime}\right)$ induces the sequence (5.5), the triangle $\left(\hat{y}_{1}^{\prime}, \pi_{2}^{\prime}, \delta_{2}^{\prime}\right)$ induces the sequence (5.11). This suffices to finish the proof of the theorem. To see this define $\hat{y}_{i}$ as $\phi_{i}^{*}\left(\hat{y}_{i}^{\prime}\right)$, where $\phi_{i}$ are the fill-in maps between the relevant exact triangles. Since $\left(\delta_{i}^{\prime}\right)^{*} \hat{y}_{i}^{\prime}=$ $Q_{t} \Sigma^{2 i d, i d} \iota$, by commutativity of the square, we conclude that $\delta_{i}^{*} \hat{y}_{i}=Q_{t} \Sigma^{2 i d, i d} \iota$ which is the statement of Theorem 5.1 since we can take $y_{i}:=\sigma\left(\hat{y}_{i}\right)$, where $\sigma$ is the canonical suspension isomorphism $\sigma: H^{*-1, *}\left(\Sigma^{-1,0} \mathbf{E}, \mathbb{Z} / q\right) \cong H^{*, *}(\mathbf{E}, \mathbb{Z} / q)$.

\subsection{Remarks on the condition (2)}

We end this manuscript with some remarks about the conditions we have assumed to prove Theorem 5.1. From [16, Theorem 1.4] it follows that $\mathcal{B}^{* *}=0$ if $\operatorname{char}(k)=$ 0 . In that case the conditions are trivially verified, but for such fields we have resolution of singularities and the spectra $\boldsymbol{\Phi}_{i}$ have already been constructed in [2]. 
It is not necessary for $\mathcal{B}^{* *}$ to vanish in order for the condition (2) to be satisfied. We are going to prove that a reasonable assumption such as the existence for all the stable motivic cohomology operations in $\mathcal{A}_{m}^{* *}$ of some "Cartan formula" respecting the splitting $\mathcal{A}_{m}^{* *} \cong \mathcal{A}^{* *} \oplus \mathcal{B}^{* *}$ suffices for the vanishing of the Margolis homology of $\mathcal{A}_{m}^{* *}$, thus of $\mathcal{B}^{* *}$, as well. A Cartan formula is a formula that describes the way a cohomology operation $\theta$ acts on the cup product of two cohomology classes of a space. It is known that the cohomology operations lying in $\mathcal{A}^{* *}$ posses such formula, that is described explicitely in [13, Proposition 9.7].

Proposition 5.2. Let $k$ be a field for which for any $b \in \mathcal{B}^{* *}$ we have $b(x \cup y)=$ $\sum_{i} b_{i}^{\prime} x \cup b_{i}^{\prime \prime} y$. Then

(1) there exists a left $\mathcal{A}^{* *}$ module isomorphism $h: \mathcal{A}_{m}^{* *} \rightarrow \mathcal{A}^{* *} \otimes_{H^{* *}} C$, where $C$ is the left $H^{* *}$ module $H^{* *} \otimes_{\mathcal{A}^{* *}} \mathcal{A}_{m}^{* *}$;

(2) $H M\left(\mathcal{A}_{m}^{* *}, Q_{t} \cdot\right)=H M\left(\mathcal{A}_{m}^{* *}, \cdot Q_{t}\right)=0$, thus $H M\left(\mathcal{B}^{* *}, Q_{t} \cdot\right)=H M\left(\mathcal{B}^{* *}, \cdot Q_{t}\right)=$ 0 .

Proof. We prove that statement (1) implies (2). Let $b \in \mathcal{A}_{m}^{* *}$ be $b=a \otimes c$ and assume that $Q_{t} b=Q_{t}(a \otimes c)=\left(Q_{t} a\right) \otimes c=0$. We see that this implies that $Q_{t} a=0$. In [2, Theorem 9], $H M\left(\mathcal{A}^{* *}, Q_{t} \cdot\right)$ has been shown to vanish, therefore $a=Q_{t} a^{\prime}$, and $b=Q_{t}\left(a^{\prime} \otimes c\right)$, thus $H M\left(\mathcal{A}_{m}^{* *}, Q_{t} \cdot\right)=0$. To prove that $H M\left(\mathcal{A}_{m}^{* *}, Q_{t}\right)=0$ we endow $\mathcal{A}_{m}^{* *}$ of an Hopf algebroid structure over $H^{* *}$ by setting $\Delta_{\mathcal{A}_{m}^{* *}}(b)=\sum_{i} b_{i}^{\prime} \otimes b_{i}^{\prime \prime}$, where $b_{i}^{\prime}$ and $b_{i}^{\prime \prime}$ are the classes appearing in the hypothesis of the Proposition 5.2 and $\Delta_{\mathcal{A}_{m}^{* *}}(a)=\Delta_{\mathcal{A}^{* *}}(a)$ for $a \in \mathcal{A}^{* *}$, where $\Delta_{\mathcal{A}^{* *}}$ is the comultiplication of $\mathcal{A}^{* *}$. Using the construction of [6, Section 8], we can define a (canonical) antiautomorphism $\chi: \mathcal{A}_{m}^{* *} \rightarrow \mathcal{A}_{m}^{* *}$, with the property that $\chi \circ \chi=i d_{\mathcal{A}_{m}^{* *}}$. Explicitely,

$$
\chi(b)=-b-\sum_{\left|b_{i}^{\prime}\right|,\left|b_{i}^{\prime \prime}\right| \neq(0,0)} b_{i}^{\prime} \chi\left(b_{i}^{\prime \prime}\right) .
$$

In particolar, $\left.\chi\right|_{\mathcal{A}^{* *}}$ is the canonical antiautomorphism of $\mathcal{A}^{* *}$. Suppose that $\alpha Q_{t}=$ 0 in $\mathcal{A}_{m}^{* *}$. Then $\chi\left(\alpha Q_{t}\right)=\chi\left(Q_{t}\right) \chi(\alpha)=0$. In the proof of [2, Corollary 7], it has been shown that $\chi\left(Q_{t}\right)=-Q_{t}$, thus $\chi(\alpha)=Q_{t} \beta$ because of what we proved above. Applying $\chi$ to this equality we conclude that $\alpha=\chi\left(Q_{t} \beta\right)=-\chi(\beta) Q_{t}$.

To prove the statement (5.2), we slightly modify the proof of [6, Theorem 4.4]. In the same notation, we let $K=H^{* *}, A=\mathcal{A}^{* *}$ and $B=\mathcal{A}_{m}^{* *}$. However, $\mathcal{A}_{m}^{* *}$ with $\mathcal{A}^{* *}$ acting on the left by ring multiplication is not a left $\mathcal{A}^{* *}$ module coalgebra This would be the case if $\Delta_{\mathcal{A}_{m}^{* *}}$ were a ring homomorphism, but the issue of endowing $\mathcal{A}_{m}^{* *} \otimes_{H^{* *}} \mathcal{A}_{m}^{* *}$ of a ring structure is tricky as $H^{* *}$ does not lie in the center of $\mathcal{A}_{m}^{* *}$. Anyways, we do have a submodule of $\mathcal{A}_{m}^{* *} \otimes_{H^{* *}} \mathcal{A}_{m}^{* *}$ that is a ring and its product structure is the one induced by the one on $\mathcal{A}_{m}^{* *} \otimes_{\mathbb{Z} / q} \mathcal{A}_{m}^{* *}($ i.e. $(x \otimes y) \cdot(u \otimes v)=$ $\left.(-1)^{\operatorname{deg}(y) \operatorname{deg}(u)} x u \otimes y v\right)$. This ring, denoted by $\left(\mathcal{A}_{m}^{* *} \otimes_{H^{* *}} \mathcal{A}_{m}^{* *}\right)_{r}$, contains the

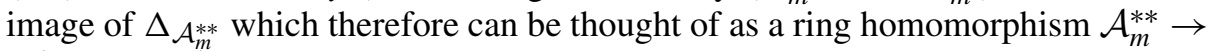
$\left(\mathcal{A}_{m}^{* *} \otimes_{H^{* *}} \mathcal{A}_{m}^{* *}\right)_{r}$. For more details on this matter, we refer to the [13, page 42 and 
Lemma 11.8]. We now follow that argument used to prove the [6, Theorem 4.4] except that we replace the compositions of the kind

$$
B \stackrel{\Delta_{B}}{\longrightarrow} B \otimes_{K} B \stackrel{\alpha \otimes \beta}{\longrightarrow} U \otimes W
$$

with the compositions

$$
\mathcal{A}_{m}^{* *} \stackrel{\Delta_{\mathcal{A}_{m}^{* *}}}{\rightarrow}\left(\mathcal{A}_{m}^{* *} \otimes_{H^{* *}} \mathcal{A}_{m}^{* *}\right)_{r} \stackrel{(\alpha \otimes \beta) \circ i}{\longrightarrow} U \otimes W
$$

where $i:\left(\mathcal{A}_{m}^{* *} \otimes_{H^{* *}} \mathcal{A}_{m}^{* *}\right)_{r} \hookrightarrow \mathcal{A}_{m}^{* *} \otimes_{H^{* *}} \mathcal{A}_{m}^{* *}$ is the canonical inclusion. For instance, the map that we wish to invert is composition $h$

$$
\mathcal{A}_{m}^{* *} \stackrel{\Delta_{\mathcal{A}_{m}^{* *}}}{\rightarrow}\left(\mathcal{A}_{m}^{* *} \otimes_{H^{* *}} \mathcal{A}_{m}^{* *}\right)_{r} \stackrel{(g \otimes \pi) \circ i}{\longrightarrow} \mathcal{A}^{* *} \otimes_{H^{* *}}\left(H^{* *} \otimes_{\mathcal{A}^{* *}} \mathcal{A}_{m}^{* *}\right)
$$

where $g$ is the retraction $\mathcal{A}_{m}^{* *} \rightarrow \mathcal{A}^{* *}$ and $\pi: \mathcal{A}_{m}^{* *} \rightarrow H^{* *} \otimes_{\mathcal{A}^{* *}} \mathcal{A}_{m}^{* *}$ is the canonical surjection $x \rightarrow 1 \otimes x$. The composition is left $\mathcal{A}^{* *}$ linear, provided that we endow $\mathcal{A}^{* *} \otimes_{H^{* *}}\left(H^{* *} \otimes_{\mathcal{A}^{* *}} \mathcal{A}_{m}^{* *}\right)$ of the "diagonal" action of $\mathcal{A}^{* *}$. This action is defined by $a(\alpha \otimes c)=\sum_{i}(-1)^{\operatorname{deg}\left(a_{i}^{\prime}\right) \operatorname{deg}(\alpha)} a_{i}^{\prime} \alpha \otimes a_{i}^{\prime \prime} c$, where $\Delta_{\mathcal{A}^{* *}} a=\sum_{i} a_{i}^{\prime} \otimes a_{i}^{\prime \prime}$. We prove that $h$ is an isomorphism of left $\mathcal{A}^{* *}$ modules by finding a left inverse $\hat{\Delta}$ which is an isomorphism in turn. The morphism $\hat{\Delta}$ is the composition

$$
\mathcal{A}^{* *} \otimes_{H^{* *}}\left(H^{* *} \otimes_{\mathcal{A}^{* *}} \mathcal{A}_{m}^{* *}\right) \stackrel{i d_{\mathcal{A}^{* *} \otimes f}^{\longrightarrow}}{\longrightarrow} \mathcal{A}^{* *} \otimes_{H^{* *}} \mathcal{A}_{m}^{* *} \stackrel{\phi}{\rightarrow} \mathcal{A}_{m}^{* *}
$$

We prove that it is an isomorphism of left $\mathcal{A}^{* *}$ modules essentially in the same way as in [6, Proposition 1.7]. In this case the map $\Delta$ of that proposition is the composition

$$
\mathcal{A}_{m}^{* *} \stackrel{\Delta_{\mathcal{A}_{m}^{* *}}}{\longrightarrow}\left(\mathcal{A}_{m}^{* *} \otimes_{H^{* *}} \mathcal{A}_{m}^{* *}\right)_{r} \stackrel{\left(i d_{\mathcal{A}_{m}^{* *}} \otimes \pi\right) \circ i}{\longrightarrow} \mathcal{A}_{m}^{* *} \otimes_{H^{* *}}\left(H^{* *} \otimes_{\mathcal{A}^{* *}} \mathcal{A}_{m}^{* *}\right)
$$

where the left $\mathcal{A}^{* *}$ action on the latter module is just on $\mathcal{A}_{m}^{* *}$ factor on the left.

\section{References}

[1] S. BoRghESI, "Algebraic Morava $K$-theories and the Higher Degree Formula", PhD Thesis, Northwestern University, 2000, http://www.math.uiuc.edu/K-theory/0412/.

[2] S. BorghesI, Algebraic Morava K-theories, Invent. Math. 151 (2003), 381-413.

[3] A. A. Elmendorf, I. Kriz, M. A. Mandell and J. P. MaY, "Rings, Modules, and Algebras in Stable Homotopy Theory", Mathematical Surveys and Monographs, Vol. 47, American Mathematical Society, Providence, RI, 1997, xii-249.

[4] J. F. JARDINE, Motivic symmetric spectra, Doc. Math. 5 (2000), 445-552.

[5] J. Milnor, The Steenrod Algebra and its Dual, Ann. of Math. (2) 67 (1958), 150-171.

[6] J. Milnor and J. MoORE, On the structure of Hopf algebras, Ann. of Math. (2) 81 (1965), 211-264. 
[7] J. Milnor and J. StashefF, 'Characteristic Classes”, Princeton University Press and University of Tokyo Press, Princeton, New Jersey, 1974.

[8] F. Morel and V. Voevodsky, $\mathbb{A}^{1}$-homotopy theory of schemes, Publ. Math. Inst. Hautes Étude Sci. 90 (1999), 45-143.

[9] P. HU, "S-modules in the Category of Schemes", Mem. Amer. Math. Soc., Vol. 161, 2003, no. 767 , viii- 125 .

[10] A. SUSLIN and V. VoeVODSKY, Bloch-Kato conjecture and motivic cohomology with finite coefficients, In: "The arithmetic and geometry of algebraic cycles" (Banff, AB, 1998), 117189, Nato Sci. Ser. C Math. Phys. Sci., Vol. 548, Kluwer Acad. Publ., Dordrecht, 2000.

[11] R. M. SwITZER, "Algebraic Topology-Homotopy and Homology", Springer-Verlag, New York-Heidelberg, 1975, xii-526.

[12] V. Voevodsky, Lectures on motivic cohomology 2000/2001 (written by Pierre Deligne). http://www.math.uiuc.edu/K-theory/0527/

[13] V. VOEVODSKY, Reduced power operation in motivic cohomology, Publ. Math. Inst. Hautes Étude Sci. 98 (2003), 1-57.

[14] V. VoeVods Ky, Motivic cohomology with $\mathbb{Z} / 2$ coefficients, Publ. Math. Inst. Hautes Étude Sci. 98 (2003), 59-104.

[15] V. VOEVODSKY, On motivic cohomology with $\mathbb{Z} /$ l-coefficients, preprint, http://www.math.uiuc.edu/K-theory/0639/.

[16] V. VoevodsKy, Motivic Eilenberg-MacLane spaces, http://www.math.uiuc.edu/K-theory/0864/.

Università degli Studi di Milano Bicocca Dipartimento di Matematica e Applicazioni via Cozzi, 53

20125 Milano, Italia 